\title{
A Unified Framework for Modeling Hysteresis in Ferroic Materials
}

\author{
Ralph C. Smith \\ Department of Mathematics \\ Center for Research in Scientific Computation \\ North Carolina State University \\ Raleigh, NC 27695 \\ rsmith@eos.ncsu.edu \\ Marcelo Dapino \\ Department of Mechanical Engineering \\ The Ohio State University \\ Columbus, OH 43210 \\ dapino.1@osu.edu
}

\author{
Stefan Seelecke \\ Mechanical and Aerospace Engineering \\ Center for Research in Scientific Computation \\ North Carolina State University \\ Raleigh, NC 27695 \\ stefan_seelecke@ncsu.edu \\ Zoubeida Ounaies \\ Department of Mechanical Engineering \\ Virginia Commonwealth University \\ Richmond VA 23284-3015 \\ zounaies@vcu.edu
}

\begin{abstract}
This paper addresses the development of a unified framework for quantifying hysteresis and constitutive nonlinearities inherent to ferroelectric, ferromagnetic and ferroelastic materials. Because the mechanisms which produce hysteresis vary substantially at the microscopic level, it is more natural to initiate model development at the mesoscopic, or lattice, level where the materials share common energy properties along with analogous domain structures. In the first step of the model development, Helmholtz and Gibbs energy relations are combined with Boltzmann theory to construct mesoscopic models which quantify the local average polarization, magnetization and strains in ferroelectric, ferromagnetic and ferroelastic materials. In the second step of the development, stochastic homogenization techniques are invoked to construct unified macroscopic models for nonhomogeneous, polycrystalline compounds exhibiting nonuniform effective fields. The combination of energy analysis and homogenization techniques produces low-order models in which a number of parameters can be correlated with physical attributes of measured data. Furthermore, the development of a unified modeling framework applicable to a broad range of ferroic compounds facilitates material characterization, transducer development, and model-based control design. Attributes of the models are illustrated through comparison with piezoceramic, magnetostrictive and shape memory alloy data and prediction of material behavior.
\end{abstract}

Keywords: Ferroic materials, unified models, hysteresis, constitutive nonlinearities 


\section{Introduction}

Ferroelectric, ferromagnetic and ferroelastic materials share a number of mesoscopic and macroscopic attributes including the formation of analogous domain structures and the presence of hysteresis and constitutive nonlinearities as illustrated in Figure 1. While hysteresis and constitutive nonlinearities are inherent to all presently employed ferroic compounds, the degree and severity of these effects can often be mitigated by restricting drive levels, employing appropriate drive electronics, or incorporating feedback loops in transducers. In many applications, however, hysteresis and material nonlinearities are unavoidable and must be incorporated in models and subsequent control designs to achieve the full capabilities of the materials. To illustrate, we consider the behavior of certain representative ferroelectric, ferromagnetic and ferroelastic materials employed in present high performance transducer applications.

Piezoceramic actuators are employed for nanoscale positioning due to their high set point accuracy and broadband capability. In present atomic force microscope (AFM) or scanning tunneling microscopic (STM) designs, PID or robust control laws are employed to mitigate hysteresis in the relation between input fields or voltages and strains generated by the device [7, 28, 87, 88, 89, 90].

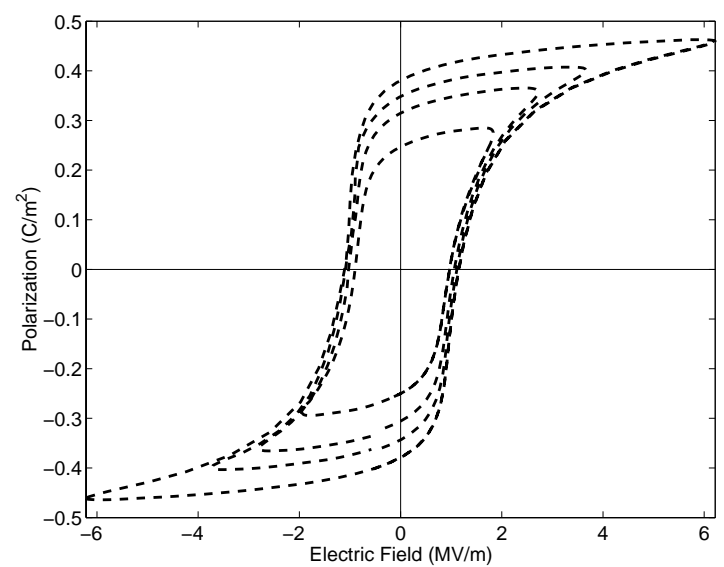

(a)

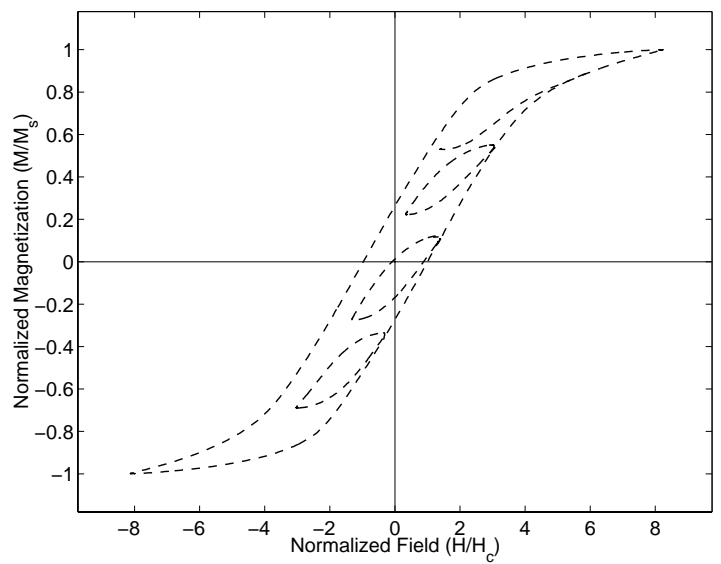

(b)

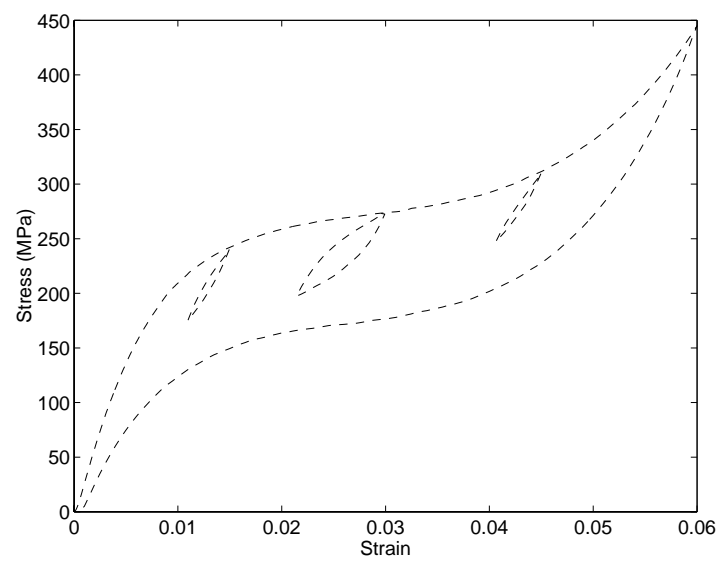

(c)

Figure 1. Hysteresis and constitutive nonlinearities exhibited by various ferroic compounds. (a) PZT5A data, (b) Terfenol-D data, and (c) NiTi data. 
This is effective for low scan rates and has led to the phenomenal success of the devices. However, at the higher scan rates required for mass product diagnostics (e.g., quality evaluation of semiconductor chips) or real-time monitoring of biological processes (e.g., observation of protein folding dynamics), noise to data ratios and diminishing high pass characteristics of control filters preclude the sole use of feedback control laws to mitigate hysteresis, and control designs utilizing model-based inverse compensators are under investigation [24, 25, 111, 124]. In other applications utilizing piezoceramic actuators, the use of charge or current controlled amplifiers can essentially eliminate hysteresis $[64,65,66,67,68]$. However, this mode of operation can be prohibitively expensive as compared with the more commonly employed voltage control amplifiers, and current control is ineffective if maintaining DC offsets as required for numerous applications - e.g., the $x$-stage in an AFM must hold a specified position while a sweep is performed in the $y$-stage.

In magnetic materials, significant research is focused on the development of compositions and compounds which maximize performance specifications such as output strains and blocked forces while minimizing hysteresis. This has motivated the development of new rare earth transducer compounds, such as Galfenol [21, 22], as well as ferromagnetic shape memory alloy (FSMA) compounds $[20,37,54,78,116]$. However, the materials with the highest work density metrics still exhibit significant hysteresis and constitutive nonlinearities which must be accommodated in transducer design. Moreover, for other technologies including magnetic recording, a high degree of hysteresis is actually required to provide the history required for the application.

In the class of ferroelastic materials, shape memory alloys (SMA) are being increasingly considered for civil, aeronautic, aerospace and industrial applications which require significant passive damping or utilize the high work output densities exhibited by the materials. Because the energy dissipated by ferroic materials is proportional to the area of the hysteresis loop, pseudoelastic operating regimes which maximize hysteresis are required when employing SMA as tendons to attenuate earthquake or wind-induced vibrations in buildings or as fibers to eliminate vibrations in articulated antennas or membrane mirrors - see [4,33,34,35, 121] for recent civil applications and [91, 92] for details regarding the modeling of hysteresis-induced damping behavior. The utilization of temperatureinduced phase transitions to provide actuator capabilities is under intense investigation in the context of microelectromechanical systems (MEMS), thin film SMAs, and microactuator applications since surface area to volume ratios in these geometries promote rapid cooling and hence high frequency drive capabilities. For example, Ho et al. report on a thin film micropump capable of operating at $300 \mathrm{~Hz}$ [46]. Additional details regarding thin film SMA applications can be found in [39, 61, 69] and discussion regarding the development of SMA-based microgrippers relevant for microrobotics and microassembly is provided in [59, 84, 85]. To achieve both the stress-induced damping capabilities and temperature-induced actuator capabilities of SMA devices, it is necessary to quantify the inherent hysteresis and constitutive nonlinearities in a manner feasible for transducer design and real-time control implementation.

Finally, there is an increased focus on the design of hybrid transducers which utilize complementary properties of constituent materials. For example, the drive characteristics and $90^{\circ}$ phase shift between Terfenol-D and PZT or PMN is being utilized to construct hybrid transducers having improved energy efficiencies and frequency bandwidths $[15,16,36,55]$. However, the hysteresis and nonlinearities inherent to the constituent materials must be accommodated to achieve optimal performance with hybrid designs.

Hence from both the fundamental perspective of material characterization and future material development, and the practical perspective of transducer design and model-based control development, it is advantageous to develop unified frameworks for quantifying hysteresis and material nonlinearities inherent to ferroelectric, ferromagnetic and ferroelastic materials. Material development and characterization necessitate a high degree of accuracy whereas the practical requirements of trans- 
ducer design and real-time control implementation limit the complexity of models and favor models which can be efficiently constructed and updated to accommodate changing operating conditions. In this paper, we employ a multiscale development to provide a unified framework for quantifying the hysteresis and constitutive nonlinearities inherent to a broad range of ferroic compounds. The goal is to provide sufficient accuracy for material characterization while maintaining a complexity level which facilitates transducer and control design in addition to real-time control implementation.

As summarized in Table 1, ferroelectric, ferromagnetic and ferroelastic materials share a number of common traits including the presence of multiple domains, which are separated by domain boundaries or walls, and field-induced phase transitions in the vicinity of the Curie temperature $T_{c}$. A unified analysis of these shared properties dates back at least to the work of Nye [77] and, in 1970, Aizu presented a unified treatment of certain symmetry properties of ferroelectric, ferromagnetic and ferroelastic compounds which he collectively referred to as ferroic compounds [5]. Shared attributes of ferroic compounds have subsequently been exploited by a number of researchers when categorizing properties of the materials [76, 109, 120].

The physical mechanisms which produce hysteresis and constitutive nonlinearities in ferroelectric, ferromagnetic and ferroelastic compounds differ significantly at the microscopic scales. In ferroelectric materials, hysteresis is partially attributed to dipole switching and domain wall losses whereas moment rotation and domain wall losses produce hysteresis in ferromagnetic compounds. While a number of analogies between hysteresis mechanisms in ferroelectric and ferromagnetic compounds can be made based on shared domain properties of the materials, the actual molecular mechanisms and scales on which domain properties occur, differ substantially. Furthermore, hysteresis in shape memory alloys is due to phase transitions between austenite and martensite variants. Hence the construction of unified energy-based models at microscopic scales is not considered feasible.

At the lattice, or mesoscopic, and macroscopic scales, however, shared domain and energy properties can be utilized to construct unified models for a broad range of ferroic materials. In the first step of the present development, Helmholtz and Gibbs energy relations are derived at the lattice level and employed in Boltzmann theory to obtain evolution equations which quantify the local average polarization, magnetization and strains in ferroelectric, ferromagnetic and ferroelastic materials. For homogeneous, single crystal compounds, these local relations can be extended throughout the material to obtain macroscopic constitutive relations which quantify the bulk material behavior. In the second step of the model development, stochastic homogenization techniques are employed to incorporate material nonhomogeneities, polycrystallinity, and nonuniform effective fields found in typical ferroic compounds. The resulting macroscopic models are low order, and hence efficient to implement, with parameters that can be correlated with properties of the data. Furthermore, the unified models guarantee the closure of biased minor loops and enforce the 'deletion' property in quasistatic

\begin{tabular}{l|l|l}
\hline Ferroelectric & Ferromagnetic & Ferroelastic \\
\hline Polarization & Magnetization & Strain \\
Electric field & Magnetic field & Stress \\
Paraelectric phase & Paramagnetic phase & Austenite phase \\
Ferroelectric phase & Ferromagnetic phase & Martensite phase \\
Ferroelectric domain walls & Bloch or Neel walls & Boundaries between variants \\
Devonshire theory & Mean field (Weiss) theory & Landau theory \\
Micromechanical ferroelectric theory & Micromagnetic theory & Ginzburg-Landau theory \\
\hline
\end{tabular}

Table 1. Analogies between physical properties of ferroic materials. 
drive regimes, and incorporate various temperature dependencies and relaxation mechanisms. The resulting unified modeling framework, which has its genesis in the SMA theory of Achenbach and Müller $[1,2]$, thus quantifies a wide range of phenomena common to ferroelectric, ferromagnetic and ferroelastic compounds and pertinent to transducer designs which exploit the unique capabilities of these materials.

To place this framework in perspective, it is useful to compare it with existing energy-based and phenomenological methodologies used to quantify hysteresis in ferroic compounds. We refer the reader to $[18,48,51,52,62,86,108,110]$ for a summary of nonlinear and hysteresis models for ferroelectric and piezoelectric compounds, $[17,29,30,31,56,58,63,71]$ for ferromagnetic materials, and $[10,40,49,50,80,81,82,99]$ for an overview of models for shape memory alloys. We summarize next investigations focused on the development of unified models for ferroic compounds.

The theory in [109] exploits the shared domain structure of ferroic materials to construct unified hysteresis models. In that development, statistical mechanics tenets are invoked to first construct anhysteretic models for idealized materials devoid of pinning sites. Electrostatic, magnetostatic and elastic energy relations are then employed to quantify the reversible and irreversible energy required to translate domain walls in the materials. The resulting models are low-order but have the disadvantage that it is difficult to enforce closure of biased minor loops without a priori knowledge of turning points. We point out that the asymptotic models derived in the present framework through statistical mechanics principles are the Ising model employed in the theory of [109] to quantify the equilibrium anhysteretic behavior of ferromagnetic and ferroelectric materials.

A variety of phenomenological techniques have also been developed to construct unified hysteresis models for ferroic compounds. In the theory developed by Soukhojak and Chiang [115], phenomenological principles are used to construct rheological models which quantify the time and frequency-dependent behavior of a range of ferroic materials. Preisach techniques have also been employed for characterizing hysteresis and nonlinearities in numerous ferroic compounds due to their generality and rigorous mathematical foundation [3, 8, 11, 41, 71, 60, 83, 119]. The generality of Preisach models also constitutes a weakness in the technique since it is difficult to incorporate known physics or properties of the data when determining parameters in the models. As discussed in $[31,63]$, extensions to classical Preisach theory are also required when incorporating reversible effects and temperature and frequency dependencies, and relaxing congruency properties to model the incongruent behavior exhibited by materials. In [112], it is illustrated that the framework presented here provides an energy basis for extended Preisach models with four important differences. (i) The energy derivation yields physical parameters which can be correlated with properties of the data this facilitates model construction and updating to accommodate changing system inputs. (ii) The temperature dependencies and relaxation mechanisms are incorporated in the basis, or hysterons, rather than in the densities, measures or parameters - this eliminates the necessity of lookup tables or vector-valued parameters when employing the models in variable temperature or certain dynamic regimes. (iii) The model does not erroneously enforce congruency and hence does not need to be modified to achieve the noncongruency exhibited in a number of operating regimes. (iv) The model automatically incorporates reversibility through the energy derivation of the hysterons and hence does not require modification to accommodate low drive regimes.

In Section 2, we develop mesoscopic energy relations, differential equations quantifying the evolution of phase fractions, and asymptotic relations characterizing the average local polarization, magnetization and strains in ferroelectric, ferromagnetic and ferroelastic compounds. The theory is extended in Section 3 to nonhomogeneous, polycrystalline compounds exhibiting nonuniform effective fields by considering parameters such as coercive fields and relative stresses to be manifestations of underlying distributions. Attributes of the unified models are then illustrated in Section 4 through comparison with PZT5A, Terfenol-D and NiTi data as well as prediction of material behavior. 


\section{Mesoscopic Models}

We summarize here the development of mesoscopic models derived through the construction of appropriate Helmholtz and Gibbs energy relations at the lattice level with Boltzmann theory used to quantify the probability of achieving various energy states. When combined with differential equations which quantify temperature changes within the materials, this yields a coupled set of evolution equations quantifying the local average polarization, magnetization and strains in ferroelectric, ferromagnetic and ferroelastic compounds. For homogeneous, single crystal compounds having uniform fields or stresses, these local relations apply throughout the material and hence provide macroscopic models which characterize hysteresis and constitutive nonlinearities. These local, mesoscopic relations are extended in Section 3 to accommodate the effects of polycrystallinity, material defects, inclusions, nonhomogeneities, and variable effective fields and stresses. To simplify the discussion, we develop both the mesoscopic and macroscopic polarization, magnetization and strain relations in the context of piezoceramic, magnetostrictive and shape memory compounds. However, the theory is sufficiently general to encompass a broad range of ferroelectric, ferromagnetic and ferroelastic materials.

To provide a general framework for formulating the unified models, we consider an order parameter $e$ and external fields $\varphi$ that are thermodynamically conjugate to $e$. For ferroelectric, ferromagnetic and ferroelastic materials, natural choices for $e$ are respectively the polarization $P$, magnetization $M$, and strains $\varepsilon$. The corresponding external fields $\varphi$ are taken to be the electric field $E$, magnetic field $H$, and stresses $\sigma$. For temperatures $T$, we denote Helmholtz energy relations by $\psi(e, T)$ and Gibbs energy relations by $G(e, \varphi, T)$.

In the absence of applied fields, the Helmholtz relations provide a natural measure of energy and, under the assumption of differentiability, thermodynamic equilibria are provided by the necessary condition

$$
\frac{\partial \psi}{\partial e}=0
$$

In the presence of an applied field, the Gibbs relation

$$
G(e, \varphi, T)=\psi(e, T)-\varphi e
$$

is used to quantify the total energy, and equilibrium states are specified by the necessary conditions

$$
\frac{\partial G}{\partial e}=0 \quad, \quad \frac{\partial^{2} G}{\partial e^{2}}>0 .
$$

The relation (3) can be interpreted as providing conditions under which the order parameter adjusts to balance the internal energy with work due to the applied field. As will be noted in subsequent discussion, the conditions (3) also provide asymptotic conditions specifying the local polarization, magnetization and strains at the lattice level as well as macroscopic constitutive relations if electromechanical or magnetomechanical coupling is included in the Gibbs energy relation.

\subsection{Piezoceramic Materials}

Presently employed piezoceramic compounds are comprised of $\mathrm{PbTi}_{1-x} \mathrm{O}_{3}$ and $\mathrm{PbZr}_{x} \mathrm{O}_{3}$ with $x$ chosen to optimize electromechanical coupling. To simplify the discussion, we focus on $\mathrm{PbTiO}_{3}$ to motivate the construction of appropriate energy relations. At temperatures $T$ above the Curie temperature $T_{c}, \mathrm{PbTiO}_{3}$ exhibits a cubic form, whereas it has the tetragonal form depicted in Figure 2a for $T<T_{c}$. A corresponding potential or Helmholtz energy profile associated with the position of the Ti cation along the $x_{3}$ axis is depicted in Figure 2b. In the presence of an applied 


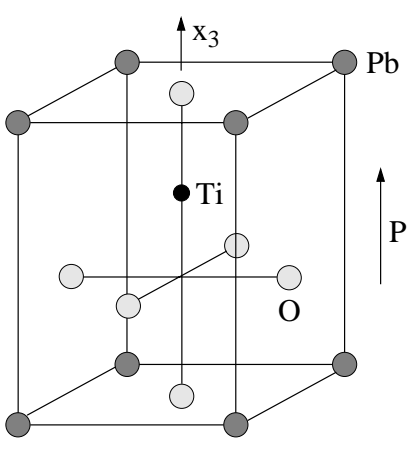

(a)

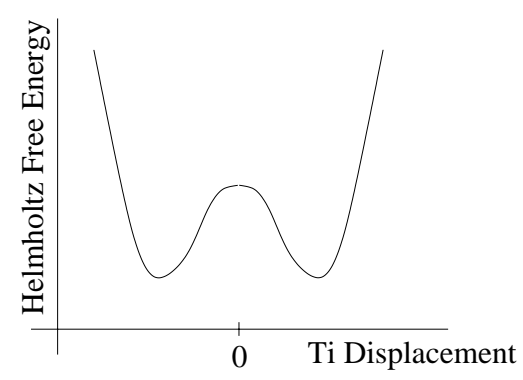

(b)

Figure 2. (a) Tetragonal structure of $\mathrm{PbTiO}_{3}$ for $T<T_{c}$ and resulting spontaneous polarization. (c) Helmholtz energy as a function of Ti position along the $x_{3}$-axis.

field $E$, the energy landscape distorts in the manner depicted in Figure 3. In the absence of thermal energy, the local minimum associated with the stable equilibrium disappears when the field reaches the local coercive field value $E_{c}$ and it becomes energetically favorable for the Ti cation to change configurations. This produces a dipole switch and subsequent hysteresis in the relation between $E$ and the local average polarization $\bar{P}$. Whereas other mechanisms, including thermal activation, contribute to the hysteresis and constitutive nonlinearities exhibited by piezoceramic materials, this source of dipole switching helps motivate appropriate formulations for the Helmholtz and Gibbs energy relations.

\subsubsection{Helmholtz and Gibbs Energy Relations}

Following the development in [114], we consider a uniform lattice of volume $V$ and mass $\nu$ having $N$ cells. We also make the assumption that each cell has two possible orientations $s_{i}= \pm 1$ and dipole moment $p$. We let $\Phi_{0}$ denote the energy required to reorient a single dipole when the lattice is completely ordered.

The Helmholtz energy for the lattice is

$$
\psi=U-S T
$$

where $U$ and $S$ respectively denote the internal energy and entropy. As detailed in [114], the combination of mean field theory and classical statistical mechanics arguments yields the Helmholtz energy relation

$$
\begin{aligned}
\psi(P, T) & =\frac{\Phi_{0} N}{2 V}\left[1-\left(P / P_{s}\right)^{2}\right]+\frac{T k N}{2 V P_{s}}\left[P \ln \left(\frac{P+P_{s}}{P_{s}-P}\right)+P_{s} \ln \left(1-\left(P / P_{s}\right)^{2}\right)\right] \\
& =\frac{E_{h} P_{s}}{2}\left[1-\left(P / P_{s}\right)^{2}\right]+\frac{E_{h} T}{2 T_{c}}\left[P \ln \left(\frac{P+P_{s}}{P_{s}-P}\right)+P_{s} \ln \left(1-\left(P / P_{s}\right)^{2}\right)\right] .
\end{aligned}
$$

Here $E_{h}=\frac{N \Phi_{0}}{V P_{s}}$ is a bias field, $T_{c}=\frac{\Phi_{0}}{k}$ is the Curie temperature ( $k$ is Boltzmann's constant), and $P_{s}=\frac{N p}{V}$ denotes the saturation polarization. It is illustrated in [114] that $\psi$ yields a double well potential for $T<T_{c}$ and a single well potential for $T>T_{c}$ in accordance with the transition from a ferroelectric phase to a paraelectric phase. 

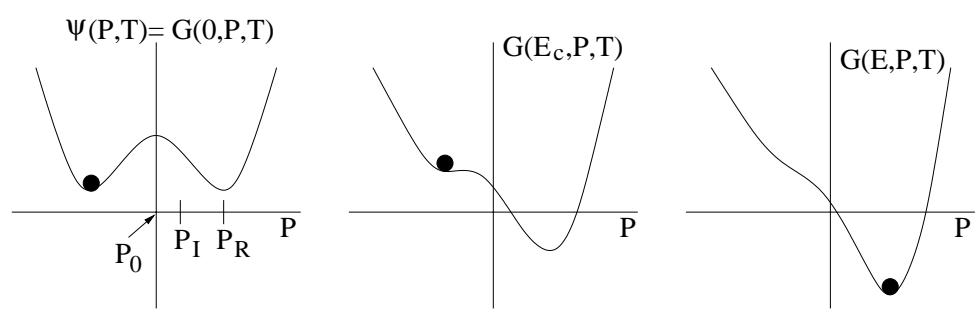

(a)
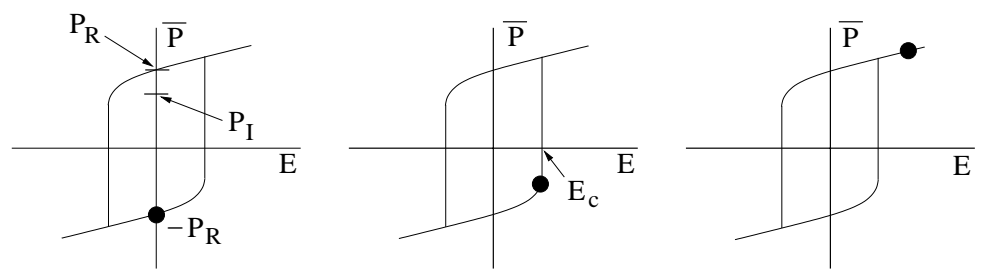

(b)

Figure 3. (a) Helmholtz energy $\psi$ and Gibbs energy $G$ for increasing fields $E$. (b) Dependence of the local average polarization $\bar{P}$ on the field $E$ at the lattice level in the presence of thermal activation.

To simplify subsequent implementation for fixed temperature regimes $T<T_{c}$, we also employ the piecewise quadratic Helmholtz relation

$$
\psi(P)= \begin{cases}\frac{1}{2} \eta\left(P+P_{R}\right)^{2} & , P \leq-P_{I} \\ \frac{1}{2} \eta\left(P-P_{R}\right)^{2} & , P \geq P_{I} \\ \frac{1}{2} \eta\left(P_{I}-P_{R}\right)\left(\frac{P^{2}}{P_{I}}-P_{R}\right) & ,|P|<P_{I}\end{cases}
$$

obtained through Taylor expansion of (4) in neighborhoods of the equilibria. The parameters $P_{R}$ and $P_{I}$ respectively denote the local polarization value at which a local minimum of $\psi$ occurs (see (1)) and the inflection point. From a physical perspective, $P_{R}$ and $\eta$ represent the local remanence value and reciprocal of the slope at saturation for the hysteron depicted in Figure 3b. Finally, we note that the local coercive field $E_{c}$ is related to $P_{R}, P_{I}$ and $\eta$ through the relation

$$
E_{c}=\eta\left(P_{R}-P_{I}\right)
$$

From the potential energy relation $U_{E}=-\mathbf{p} \cdot \mathbf{E}$, it follows from (2) that an expression for the Gibbs energy is

$$
G(P, E, T)=\psi(P, T)-E P
$$

for uniaxial lattice structures. The behavior of $G$ for an increasing field and the resulting local average polarization $\bar{P}$ are depicted in Figure 3 .

\subsubsection{Local Average Polarization}

To quantify $\bar{P}$ it is necessary to determine the fractions of dipoles $x_{+}$and $x_{-}$respectively having positive and negative orientations, the likelihoods $p_{+-}$and $p_{-+}$of switching from positive to negative, and conversely, and the expected polarization values $\left\langle P_{+}\right\rangle$and $\left\langle P_{-}\right\rangle$associated with positive and negative dipoles. In the absence of thermal activation, these quantities follow directly from the necessary condition (3) which forms a basis for subsequent asymptotic relations for $\bar{P}$. To incorporate 
measured relaxation phenomena, it is necessary to incorporate a balance of the thermal and Gibbs energies through the Boltzman relation

$$
\mu(G)=C e^{-G V / k T} .
$$

The constant $C$ is chosen to guarantee integration to unity over all admissible inputs and $k$ again denotes Boltzmann's constant. Physically, the probability $\mu(G)$ of achieving an energy level $G$ is increased for large values of the relative thermal energy $k T / V$ and diminished for small $k T / V$. Hence more dipoles will have the energy required to jump from one stable equilibrium to the other when $k T / V$ is large than when $k T / V$ is small or in the limiting case when $k T / V \rightarrow 0$.

As detailed in [114], the likelihoods $p_{+-}$and $p_{-+}$are given by

$$
p_{+-}=\frac{1}{\mathcal{T}(T)} \frac{e^{-G\left(E, P_{I}(T), T\right) V / k T}}{\int_{P_{I}(T)}^{\infty} e^{-G(E, P, T) V / k T} d P} \quad, \quad p_{-+}=\frac{1}{\mathcal{T}(T)} \frac{e^{-G\left(E,-P_{I}(T), T\right) V / k T}}{\int_{-\infty}^{-P_{I}(T)} e^{-G(E, P, T) V / k T} d P}
$$

where the formulation in terms of the inflection points $\pm P_{I}$ rather than the equilibrium value $P_{0}$ can be argued either from energy principles or asymptotic analysis. The relaxation time $\mathcal{T}$ for the material is related to the temperature and reference volume $V$ of mass $\nu$ by the relation

$$
\mathcal{T}(T)=\sqrt{\frac{2 \pi \nu V^{2 / 3}}{k T}}
$$

which can be interpreted as the reciprocal of the frequency at which dipoles attempt jumps. By integrating the product $P \mu(G)$ over all admissible polarization values and evaluating the integration constant $C$, it follows that the average polarization values can be expressed as

$$
\left\langle P_{+}\right\rangle=\frac{\int_{P_{I}}^{\infty} P e^{-G(E, P, T) V / k T} d P}{\int_{P_{I}}^{\infty} e^{-G(E, P, T) V / k T} d P} \quad, \quad\left\langle P_{-}\right\rangle=\frac{\int_{-\infty}^{-P_{I}} P e^{-G(E, P) V / k T} d P}{\int_{-\infty}^{-P_{I}} e^{-G(E, P) V / k T} d P} .
$$

The evolution of dipole fractions is quantified by the differential equations

$$
\begin{aligned}
& \dot{x}_{+}=-p_{+-} x_{+}+p_{-+} x_{-} \\
& \dot{x}_{-}=-p_{-+} x_{-}+p_{+-} x_{+}
\end{aligned}
$$

which can be simplified to

$$
\dot{x}_{+}=-p_{+-} x_{+}+p_{-+}\left(1-x_{+}\right)
$$

through the identity $x_{+}+x_{-}=1$.

With the dipole fractions, transition likelihoods, and average polarization values thus defined, the average local polarization at the lattice level is given by

$$
\bar{P}=x_{+}\left\langle P_{+}\right\rangle+x_{-}\left\langle P_{-}\right\rangle .
$$

The formulation (14) incorporates thermal relaxation through the relation (10) as well as certain temperature dependencies if the Helmholtz relation (4) is employed. While the use of (4) has been illustrated for incorporating the temperature dependence associated with certain relaxor ferroelectric compounds, more general temperature dependencies can be incorporated by modifying the piecewise quadratic relation (5) in the manner illustrated in Section 2.3 for shape memory alloys. 


\subsubsection{Thermal Evolution}

To quantify changes in temperature due to convection and conduction to surrounding media, Joule heating, and dipole switching, we employ a balance of energy to obtain the evolution equation

$$
\bar{c} \mathcal{M} \dot{T}(t)=-h_{c} \Omega\left[T-T_{E}(t)\right]-\frac{\lambda \Omega}{\ell}\left[T-T_{E}(t)\right]+J(t)-\left[h_{+} \dot{x}_{+}+h_{-} \dot{x}_{-}\right] .
$$

Here $\bar{c}, \mathcal{M}, h_{c}, \Omega, \lambda$ and $\ell$ respectively denote the specific heat for PZT, the mass of the actuator, a heat transfer coefficient, the surface area of the PZT, the thermal conductivity of the surrounding medium, and the interval over which conduction occurs [47]. The first term on the right hand side of (15) quantifies heat exchange due to convection whereas the second term incorporates potential heat loss or sources due to conduction. The term $J(t)$ characterizes temperature changes due to Joule heating. For certain operating regimes, this can be quantified by the relation

$$
J(t)=\bar{\rho}^{e} h \frac{I^{2}(t)}{\mathcal{A}}
$$

where $\bar{\rho}^{e}, h$ and $\mathcal{A}$ respectively denote the average electrical resistivity, thickness of the PZT, and cross-sectional area of the PZT [100]. To incorporate additional geometric, electromagnetic, or frequency-dependent effects, one can quantify $J(t)$ either through experimental measurements or more comprehensive models. We note that the incorporation of Joule heating mechanisms becomes increasingly important in applications requiring high drive frequencies.

The final component of (15) quantifies heat transduction due to dipole switching so that $h_{+}$and $h_{-}$are analogous to the specific enthalpies in the corresponding SMA relation (51). This relation also becomes increasingly significant for high frequency transduction. In general, validation experiments will be required to establish regimes when this latter contribution should be retained as well as operating conditions where it can be considered negligible. Finally, we point out that $\bar{c}$ and $\bar{\rho}_{e}$ are considered as averages and the specification of phase-dependent components to these coefficients may be required when quantifying the temperature changes which occur during phase transitions.

\subsubsection{Asymptotic Polarization Relations in the Absence of Thermal Activation}

For applications in which relaxation times are considered negligible, or do not constitute a significant role in transducer behavior, the local average polarization expression can be simplied significantly by considering the limiting case in which thermal fluctuations about equilibrium values are eliminated. As detailed in [114], the limiting models can be derived either through formal application of the necessary condition (3) or rigorous consideration of the limits $k T / V \rightarrow 0$ in the definition of the likelihoods $p_{+-}, p_{-+}$and average polarization values $\left\langle P_{+}\right\rangle,\left\langle P_{-}\right\rangle$. To simplify the discussion, we summarize the formal arguments here.

For the Helmholtz relation (4), enforcement of the necessary condition (3) with $\varepsilon=P$ and $\psi=E$ yields the equilibrium relation

$$
\bar{P}(E)=P_{s} \tanh \left(\frac{E+\alpha P}{a(T)}\right)
$$

where

$$
\alpha=\frac{E_{h}}{P_{s}} \quad, \quad a(T)=\frac{E_{h} T}{P_{s}},
$$

for the average local polarization. We note that (17) is exactly the Ising relation employed in [108] when quantifying the anhysteretic polarization and is equivalent through first-order terms with the Langevin relations employed in various ferroelectric and ferromagnetic models. Translates of the form $P=P_{s} \tanh \left(E \pm E_{c}\right)$ were also employed by Zhang and Rogers [123], and ridge functions 
of the form $r(x)=\tanh (x)$ are appropriate for generalized Preisach, or Krasnosel'skiǐ-Pokrovskiǐ characterizations $[8,9,60]$.

The relation resulting from (5) is slightly more complicated due to the piecewise nature of the definition. Enforcement of the necessary condition (3) yields the general relation

$$
\bar{P}=\frac{1}{\eta} E+\Delta P_{R}
$$

where $\Delta=1$ for positively oriented dipoles and $\Delta=-1$ for negative orientations. To specify $\bar{P}$ more rigorously in terms of initial dipole orientations

$$
\left[\bar{P}\left(E ; E_{c}, \xi\right)\right](0)= \begin{cases}\frac{E}{\eta}-P_{R} & , E(0) \leq-E_{c} \\ \xi & ,-E_{c}<E(0)<E_{c} \\ \frac{E}{\eta}+P_{R} & , E(0) \geq E_{c}\end{cases}
$$

and transition times

$$
\tau(t)=\left\{t \in\left(0, T_{f}\right] \mid E(t)=-E_{c} \text { or } E(t)=E_{c}\right\},
$$

we employ the Preisach notation

$$
\left[\bar{P}\left(E ; E_{c}, \xi\right)\right](t)= \begin{cases}{\left[\bar{P}\left(E ; E_{c}, \xi\right)\right](0)} & , \tau(t)=\emptyset \\ \frac{E}{\eta}-P_{R} & , \tau(t) \neq \emptyset \text { and } E(\max \tau(t))=-E_{c} \\ \frac{E}{\eta}+P_{R} & , \tau(t) \neq \emptyset \text { and } E(\max \tau(t))=E_{c}\end{cases}
$$

(e.g., see $[8,60,112])$. The dependence of the local average polarization $\bar{P}$ on the local coercive field $E_{c}$ is indicated as a prelude to the stochastic extensions to the theory developed in Section 3.

\subsection{Magnetostrictive Materials}

Field-induced magnetization changes in ferromagnetic compounds, and accompanying hysteresis, are due primarily to a combination of two mechanisms: rotation of moments and losses associated with domain wall motion $[19,27,56]$. For highly anisotropic materials, characterization of constitutive nonlinearities and hysteresis necessarily requires incorporation of the anisotropy energy when computing the energetic response of the material. For several classes of operating conditions and materials, however, models based on the restriction of spins or magnetic moments to two possible orientations, denoted $s_{i}= \pm 1$, can be developed. From a classical perspective, this assumption is valid for materials which exhibit uniaxial crystalline anisotropies or transducers in which uniaxial stresses dominate the crystalline structure. This latter category includes Terfenol-D transducers in which prestress mechanisms are employed to maintain the rod in compression and to optimize magnetomechanical coupling $[29,30]$. From a quantum perspective, the assumption of diametrically opposed spins is commensurate with the observation that two allowable spin configurations are orientations parallel and opposite to an applied field.

\subsubsection{Helmholtz and Gibbs Energies}

To construct a mesoscopic Helmholtz energy relation for ferromagnetic materials, we consider a uniform and homogeneous lattice volume $V$ of mass $\nu$ comprised of $N$ cells, each of which is assumed to contain one spin, or magnetic moment, $m$ having the orientation $s_{i}= \pm 1$. As in the ferroelectric model, we let $\Phi_{0}$ denote the energy required to reorient a single moment in a completely ordered lattice - the relation between $\Phi_{0}$ and the magnetic exchange integral $\mathcal{J}$ is detailed in [105] - and make 
the assumption that only adjacent moments interact in accordance with mean field approximation to the Ising model. As detailed in [105], formulation of the Helmholtz energy $\psi=U-S T$ in this context yields the relation

$$
\psi(M, T)=\frac{H_{h} M_{s}}{2}\left[1-\left(M / M_{s}\right)^{2}\right]+\frac{H_{h} T}{2 T_{c}}\left[M \ln \left(\frac{M+M_{s}}{M_{s}-M}\right)+M_{s} \ln \left(1-\left(M / M_{s}\right)^{2}\right)\right]
$$

where $M_{s}=\frac{N m}{V}, T_{c}=\frac{\Phi_{0}}{k}$, and $H_{h}=\frac{N \Phi_{0}}{V M_{s}}$ respectively denote the technical saturation magnetization, Curie temperature, and bias field. The initial assumption that $\Phi_{0}$ is constant implies that $H_{h}$ is constant for a uniform, homogeneous lattice. This assumption is relaxed in Section 3 where we consider statistically distributed values of $H_{h}$ to accommodate material nonhomogeneities and the effects of polycrystallinity. Finally, we note that $\psi$ yields a single well potential for $T \geq T_{c}$ and a double well potential in accordance with the transition from paramagnetic to ferromagnetic phases.

In [105], it is illustrated that Taylor expansion about the three equilibria can be employed to construct the piecewise quadratic Helmholtz relation

$$
\psi(M)= \begin{cases}\frac{1}{2} \eta\left(M+M_{R}\right)^{2} & , M \leq-M_{I} \\ \frac{1}{2} \eta\left(M-M_{R}\right)^{2} & , M \geq M_{I} \\ \frac{1}{2} \eta\left(M_{I}-M_{R}\right)\left(\frac{M^{2}}{M_{I}}-M_{R}\right) & ,|M|<M_{I}\end{cases}
$$

which facilitates implementation in fixed temperature regimes. Here $M_{I}$ and $M_{R}$ denote the inflection point and minimum of $\psi$ at which local remanence occurs - see Figure 3 for a depiction of analogous behavior for ferroelectric materials.

For variable temperature applications, one can employ either (22) or a modification of (23) in which temperature dependence is incorporated in a manner analogous to that employed in Section 2.3 for shape memory alloys. The former option automatically includes the ferromagnetic to paramagnetic phase transition whereas the latter offers advantages from the perspective of implementation.

Since the magnetostatic energy is given by $\mathcal{E}=-\mu_{0} \mathbf{m} \cdot \mathbf{H}$, where $\mu_{0}$ denotes the magnetic permeability, the uniaxial Gibbs energy can be specified either as

$$
G(M, H, T)=\psi(M, T)-\mu_{0} M H
$$

or

$$
G(M, H, T)=\psi(M, T)-M H
$$

by incorporating $\mu_{0}$ in $\psi$. We employ (24) due to its commonality with the general Gibbs relation (2) which facilitates the development of a unified modeling framework. A comparison of the Helmholtz relations (4) and (22), the piecewise quadratic relations (5) and (23), and the Gibbs energy relations (7) and (24) illustrates the commonality in the formulations for ferroelectric and ferromagnetic compounds.

\subsubsection{Average Local Magnetization}

The development of local average magnetization relations is analogous to that for ferroelectric materials and details are provided in $[105,106]$. For operating regimes in which relaxation effects are considered negligible, limiting values of the local average magnetization $\bar{M}$ can be formally obtained from the necessary condition (3). For the Helmholtz relation (22) derived from statistical mechanics principles, the condition $H=\frac{\partial \psi}{\partial M}$ yields

$$
\bar{M}=M_{s} \tanh \left(\frac{H+\alpha M}{a(T)}\right)
$$


where $\alpha=\frac{H_{h}}{M_{s}}$ and $a(T)=\frac{H_{h}(T)}{M_{s}}$. The relation (25) is an Ising model whose input is the effective field

$$
H_{e}=H+\alpha M
$$

This relation was employed as the anhysteretic component of the unified models developed in [109]. Furthermore, it is illustrated in $[108,109]$ that if one relaxes the constraint that moments have only the orientations $s_{i}= \pm 1$ and considers uniformly distributed moments, one obtains the Langevin relation $M=\mathcal{L}\left(H_{e}\right) \equiv M_{s}\left[\operatorname{coth}\left(H_{e} / a\right)-a / H_{e}\right]$ which agrees with the Ising relation $M=M_{s} \tanh \left(H_{e} / a\right)$ through first order terms - see $[19,56]$ for a derivation of the Langevin equation in the context of magnetic materials. The Langevin model $M=M_{s} \mathcal{L}\left(H_{e}\right)$, with $H_{e}$ specified by (26), is employed when quantifying the anhysteretic magnetization in the domain wall theory of Jiles and Atherton $[57,58]$ as well as the transducer models based on that theory [17, 29, 30].

The magnetization relation resulting from (24) in the absence of thermal activation is elementary in the sense that it is piecewise linear but is complicated by the fact that a history of dipole switches must be maintained to ascertain which branch of the hysteron is active. This can be accomplished via the Preisach notation

$$
\left[\bar{M}\left(H ; H_{c}, \xi\right)\right](t)= \begin{cases}{\left[\bar{M}\left(H ; H_{c}, \xi\right)\right](0)} & , \tau(t)=\emptyset \\ \frac{H}{\eta}-M_{R} & , \tau(t) \neq \emptyset \text { and } H(\max \tau(t))=-H_{c} \\ \frac{H}{\eta}+M_{R} & , \tau(t) \neq \emptyset \text { and } H(\max \tau(t))=H_{c}\end{cases}
$$

where

$$
\left[\bar{M}\left(H ; H_{c}, \xi\right)\right](0)= \begin{cases}\frac{H}{\eta}-M_{R} & , H(0) \leq-H_{c} \\ \xi & ,-H_{c}<H(0)<H_{c} \\ \frac{H}{\eta}+M_{R} & , H(0) \geq H_{c}\end{cases}
$$

denotes initial moment orientations and the switching times $\tau(t)$ are specified by (20) with switching occurring at the local coercive field values $H_{c}$ and $-H_{c}$. Heuristically, the relation (27) has the form

$$
\bar{M}=\frac{1}{\eta} H+\Delta M_{R}
$$

where $\Delta= \pm 1$ is used to delineate the upper and lower branches of the hysteron.

To incorporate thermal activation and relaxation mechanisms - e.g., when modeling magnetic after-effects of the type discussed in Chapter 20 of [19] - it is necessary to quantify the evolution of the moments fractions $x_{+}$and $x_{-}$having positive and negative spins. The notation and development is analogous to that presented in Section 2.1.2 for ferroelectric materials and details regarding the development of ferromagnetic materials can be found in $[105,106]$.

Since the probability of achieving an energy state $G$ is given by (8), the transition likelihoods $p_{+-}$and $p_{-+}$are given by

$$
p_{+-}=\frac{1}{\mathcal{T}(T)} \frac{e^{-G\left(H, M_{I}, T\right) V / k T}}{\int_{M_{I}}^{\infty} e^{-G(H, M, T) V / k T} d M} \quad, \quad p_{-+}=\frac{1}{\mathcal{T}(T)} \frac{e^{-G\left(H,-M_{I}, T\right) V / k T}}{\int_{-\infty}^{-M_{I}} e^{-G(H, M, T) V / k T} d M}
$$

where the relaxation time $\mathcal{T}$ is specified by (10). The expected magnetization values $\left\langle M_{+}\right\rangle$and $\left\langle M_{-}\right\rangle$, due to positively and negatively oriented moments, are specified by

$$
\left\langle M_{+}\right\rangle=\frac{\int_{M_{I}}^{\infty} M e^{-G(H, M, T) V / k T} d M}{\int_{M_{I}}^{\infty} e^{-G(H, M, T) V / k T} d M} \quad, \quad\left\langle M_{-}\right\rangle=\frac{\int_{-\infty}^{-M_{I}} M e^{-G(H, M) V / k T} d M}{\int_{-\infty}^{-M_{I}} e^{-G(H, M) V / k T} d M}
$$


which are analogous to the ferroelectric relations (11). Finally the average local magnetization $\bar{M}$, which incorporates thermal relaxation mechanisms, is given by

$$
\bar{M}=x_{+}\left\langle M_{+}\right\rangle+x_{-}\left\langle M_{-}\right\rangle
$$

where the moment fraction $x_{+}$is specified by the differential equation (13) and $x_{-}=1-x_{+}$. In [105], it is illustrated that the relations (27) for materials with negligible relaxation characteristics can also be rigorously derived from (30), (31) and (32) by considering the limiting case $k T / V \rightarrow 0$.

\subsubsection{Thermal Evolution}

Temperature changes in magnetostrictive transducer materials are due to several mechanisms including ohmic heating in the solenoid, which is transmitted to the Terfenol-D rod through conduction, Joule heating due to eddy currents, and potential internal heating due to the reorientation of moments. To accommodate future designs, we also incorporate possible convection mechanisms analogous to those considered for piezoceramic and shape memory alloy transducers.

To model the temperature transition in the magnetic material, we employ the notation of Section 2.1.3 and let $\bar{c}, h_{c}, \mathcal{M}, \Omega, \lambda, \ell$ and $T_{E}(t)$ respectively denote the specific heat, a convection coefficient, the mass of the Terfenol-D rod, the surface area of the material, the thermal conductivity of the solenoid, the path length of conduction, and the time varying temperature of the adjacent environment. An energy balance then yields the differential equation

$$
\bar{c} \mathcal{M} \dot{T}(t)=-\Omega\left[h_{c}+\lambda / \ell\right]\left[T-T_{E}(T)\right]+J(t)-\left[h_{+} \dot{x}_{+}+h_{-} \dot{x}_{-}\right]
$$

governing the temperature evolution. A comparison of (33) with its ferroelectric counterpart (15) reveals that the general temperature relations are identical.

To quantify the conductive and convective contributions, it is necessary to specify $T_{E}(t)$ either experimentally or through additional models. For example, a fully coupled thermal model for a Terfenol-D transducer would require the measurement or characterization of ohmic heating in the solenoid and heat transmission through the device to specify $T_{E}(t)$. Similarly, the Joule component $J(t)$ can be determined either empirically or though eddy current relations. Finally, the last term in (33) quantifies potential changes in temperature due to the reorientation of moments. The relevance of this term for specific operating conditions should be established through validation experiments.

\subsection{Shape Memory Alloys}

Shape memory alloys exhibit a number of unique features which are being targeted for present and projected applications $[69,99,101]$. Following a plastic deformation at low temperatures, the materials will recover their original shape upon the application of heat - this constitutes the shape memory effect which gives the materials their name. At higher temperatures, reversible deformations up to $10 \%$ can be obtained under nearly constant loads which gives the materials superelastic capabilities. These deformation properties are commonly termed quasiplastic at low temperatures and pseudoelastic at higher temperatures with the latter illustrated by the SMA data plotted in Figure 1c. All of these characteristics are due to temperature or load induced phase transformations at the lattice level and it is at this scale that we initiate model development. Most initial applications utilizing SMA compounds were focused on polycrystalline NiTi wires whereas NiTi films are under present investigation to support MEMs applications and to improve response times through increased surface to volume ratios. For uniaxial loads, uniaxial models suffice for both regimes and are the focus of this discussion. Hence we focus on characterizing the evolution of austenite $A$ and the martensite twins $M^{+}$and $M_{-}$in SMA lattice layers. 
Following the theory developed in $[69,79,93,98,99]$, we treat a lattice volume $V$ of mass $\nu$ as the fundamental element in the model and let $x_{A}(t), x_{+}(t)$ and $x_{-}(t)$ respectively denote the volume fraction of $A, M^{+}$and $M^{-}$layers in the SMA. The phase fractions constitute internal variables which necessarily satisfy the conservation relation

$$
x_{A}+x_{+}+x_{-}=1
$$

over all time. To consolidate notation, we let $\alpha$ generically refer to the $A, M^{+}$and $M^{-}$variants so that the relation (34) can be reformulated as

$$
\sum_{\alpha} x_{\alpha}(t)=1
$$

\subsubsection{Helmholtz and Gibbs Energies}

The formulation of Helmholtz and Gibbs energy relations is common to a number of SMA theories (e.g., see $[53,69,99,101]$ and the references cited therein), and differences between the theories arise in the manner through which energy relations are defined and used to construct macroscopic models. We employ the theory of $[69,99]$ to construct $C_{1}$ energy relations based on piecewise quadratic functions which characterize stable equilibria corresponding to the $A, M^{+}$and $M^{-}$phases.

As illustrated in Figure 4, the fundamental order parameter is the shear strain $\varepsilon$ which has the value $\varepsilon=0$ for austenite and the equilibrium value $\varepsilon=\varepsilon_{T}$ for martensite in a stress-free state. The linear moduli for martensite and austenite are respectively denoted by $Y_{M}$ and $Y_{A}$. To model the transition from stability of martensite variants at low temperatures to stability of the austenite phase at high temperatures, we employ the $C_{1}$ Helmholtz energy relation

$$
\psi(\varepsilon, T)= \begin{cases}\frac{Y_{M}}{2}\left(\varepsilon+\varepsilon_{T}\right)^{2} & , \quad \varepsilon \leq-\varepsilon_{M}(T) \\ -\frac{E_{0}(T)}{2}\left(\varepsilon+\varepsilon_{0}(T)\right)^{2}+\psi_{0}(T) & , \quad-\varepsilon_{M}(T)<\varepsilon<-\varepsilon_{A}(T) \\ \frac{Y_{A}}{2} \varepsilon^{2}+\Delta \beta(T) & , \quad|\varepsilon| \leq \varepsilon_{A}(T) \\ -\frac{E_{0}(T)}{2}\left(\varepsilon-\varepsilon_{0}(T)\right)^{2}+\psi_{0}(T) & , \quad \varepsilon_{A}(T)<\varepsilon<\varepsilon_{M}(T) \\ \frac{Y_{M}}{2}\left(\varepsilon-\varepsilon_{T}\right)^{2} & , \quad \varepsilon \geq \varepsilon_{M}(T) .\end{cases}
$$

The temperature-dependent inflection points $\varepsilon_{M}(T), \varepsilon_{A}(T)$, and their negatives, delineate the transition from convex regions, which represent stable austenite and martensite phases, to concave regions
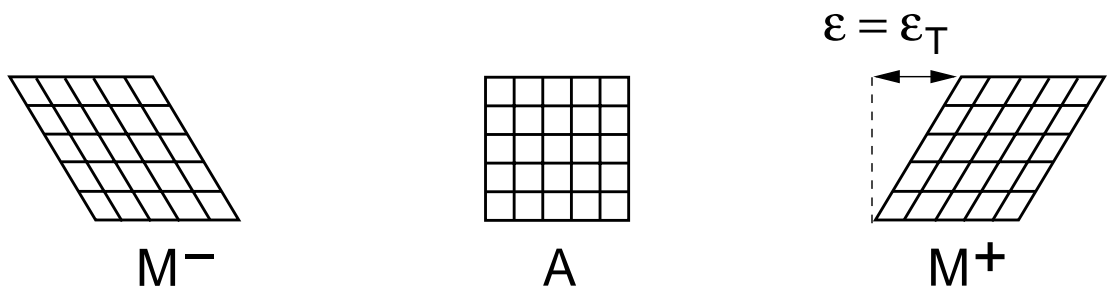

Figure 4. Lattice element exhibiting the martensite $M^{-}, M^{+}$and austenite $A$ equilibrium configurations. 


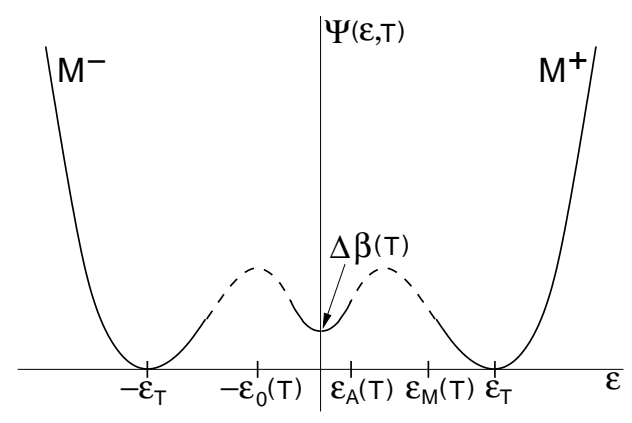

(a)

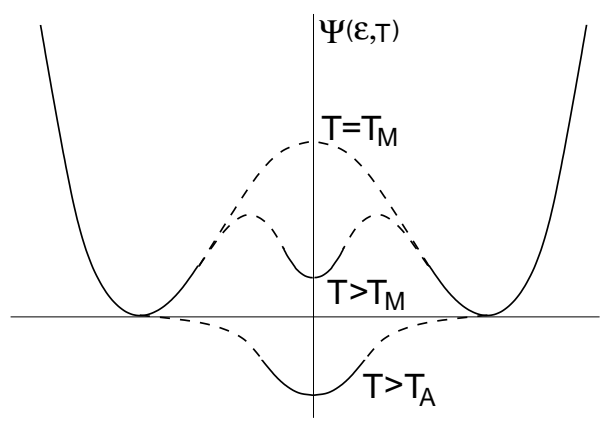

(b)

Figure 5. (a) Piecewise quadratic Helmholtz energy (35) for fixed temperature T; dashed segments represent concave, unstable regions. (b) Helmholtz energy as a function of $T$.

representing unstable states. The maxima of the concave parabolae occur at the temperaturedependent points $\left( \pm \varepsilon_{0}(T), \psi_{0}(T)\right)$, and the parameter $E_{0}(T)$ is chosen to ensure $C_{1}$ continuity. As depicted in Figure 5, the austenite minimum has the height

$$
\Delta \beta(T)=\beta_{A}(T)-\beta_{M}(T)
$$

where

$$
\beta_{\alpha}(T)=c_{\alpha}\left(T-T_{R}\right)+u_{R}-T s_{\alpha}
$$

represent chemical (nonelastic) free energies [69, 99, 101]. Here $c_{\alpha}, u_{\alpha}$ and $T_{R}$ respectively denote specific heat capacities multiplied by the material density, phase-dependent internal energy constants, and the temperature of the reference state from which energies are computed. Furthermore,

$$
S_{\alpha}=c_{\alpha} \ln \left(T / T_{R}\right)+\eta_{R}
$$

are specific entropies and $\eta_{\alpha}$ are phase-dependent entropy constants.

The Helmholtz relation (35) quantifies the energy for a layer in the absence of applied loads. To incorporate distortions in the energy landscape due to applied stresses $\sigma$, we employ the Gibbs relation

$$
G(\varepsilon, \sigma, T)=\psi(\varepsilon, T)-\varepsilon \sigma
$$

resulting from (3). In Figure 6a, we illustrate the Gibbs energy for a lattice volume $V$ at a fixed temperature $T=T_{A}$ chosen so that the material exhibits the austenite phase for $\sigma=0$. As $\sigma$ is increased, the landscape distorts until the critical stress $\sigma_{A}$ at which point the stable austenite equilibrium ceases to be a local minimum and $M^{+}$becomes the stable phase. It will remain such until the stress is decreased to a second critical value $\sigma_{M}$. Here the local martensite minimum disappears and the material returns to the austenite phase.

\subsubsection{Local Average Stress-Strain Relations}

To quantify the pseudoelastic load deformation behavior which accompanies the increase in stress through the critical value $\sigma_{A}$ and subsequent decrease through the second critical value $\sigma_{M}$, one can quantify the evolution of associated phase fractions in the absence or presence of thermal activation in a manner analogous to that summarized previously for ferroelectric and ferromagnetic compounds.

For reference volumes $V$ in which thermal activation is negligible, the local average stress-strain behavior is quantified solely by necessary conditions associated with the Gibbs energy $G$ given by (39). 


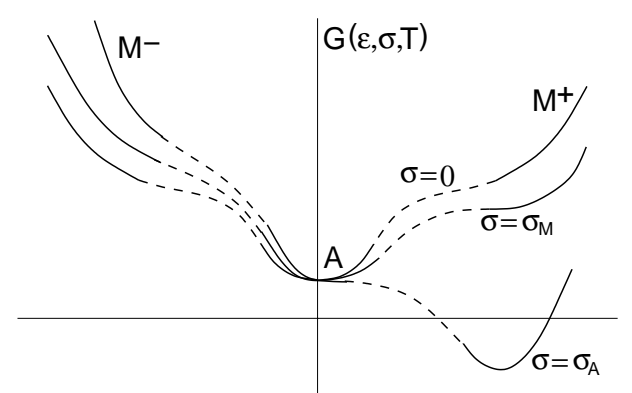

(a)

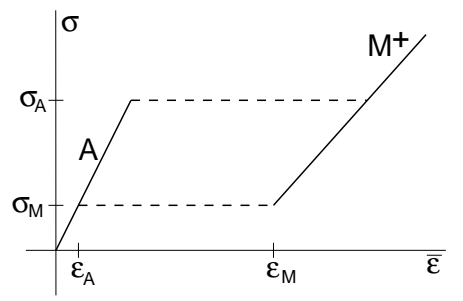

(b)

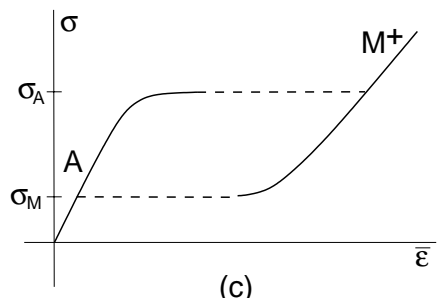

(c)

Figure 6. (a) Gibbs energy (39) for a fixed temperature $T$ in the austenite range with $\sigma=0$, $\sigma=\sigma_{A}$ and $\sigma=\sigma_{M}$. (b) Local stress-strain relation produced by the equilibrium condition (3) for fixed temperature $T$ and negligible relative thermal energy $k T / V$. (c) Local stress-strain relation in the presence of relative thermal energy $k T / V$ for fixed $T$.

From (2), it follows that the conditions

$$
\frac{\partial G}{\partial \varepsilon}=0 \quad, \quad \frac{\partial^{2} G}{\partial \varepsilon^{2}}>0
$$

must hold at stable equilibria which yields the critical stress values

$$
\begin{aligned}
& \sigma_{A}(T)=Y_{A} \varepsilon_{A}(T) \\
& \sigma_{M}(T)=Y_{M}\left[\varepsilon_{M}(T)-\varepsilon_{T}\right] .
\end{aligned}
$$

For material characterization, it is advantageous to define the temperature-dependent parameters

$$
\delta(T)=\sigma_{A}(T)-\sigma_{M}(T)
$$

which, it will be later illustrated, can be estimated from measured data. Due to the quadratic definition of the Gibbs energy, the local stress-strain behavior is linear in the absence of thermal activation, as depicted in Figure 6b. To incorporate the switching history, we employ the Preisach notation

$$
[\bar{\varepsilon}(\sigma ; \delta, \xi)](t)= \begin{cases}{[\bar{\varepsilon}(\sigma ; \delta, \xi)](0)} & , \tau(t)=\emptyset \\ \frac{\sigma}{Y_{M}}+\varepsilon_{T} & , \tau(t) \neq \emptyset \text { and } \sigma(\max \tau(t))=\sigma_{A} \\ \frac{\sigma}{Y_{A}} & , \tau(t) \neq \emptyset \text { and } \sigma(\max \tau(t))=\sigma_{M}\end{cases}
$$

to quantify the local average strains due to the positive applied loads $\sigma$ (similar expressions hold for compressive loads). Analogous to the ferroelectric definitions (19) and (20) and ferromagnetic definition (28),

$$
[\bar{\varepsilon}(\sigma ; \delta, \xi)](0)= \begin{cases}\frac{\sigma}{Y_{A}} & , \sigma(0) \leq \sigma_{M} \\ \xi & , \sigma_{M}<\sigma(0)<\sigma_{A} \\ \frac{\sigma}{Y_{M}}+\varepsilon_{T} & , \sigma(0) \geq \sigma_{A}\end{cases}
$$


and

$$
\tau(t)=\left\{t \in\left(0, T_{f}\right] \mid \sigma(t)=\sigma_{M} \text { or } \sigma(t)=\sigma_{A}\right\},
$$

respectively denote the initial phase configuration and switching times.

To quantify the local average strains $\bar{\varepsilon}$ for reference volumes in which thermal activation is significant, it is necessary to balance the Gibbs energy $G$ with the relative thermal energy $k T / V$ through the Boltzmann relation (8). Physically, large values of $k T / V$ mean that with higher probability, layers will achieve the energy required to exit a local minimum before the stress values $\sigma_{A}$ or $\sigma_{M}$ are reached. This produces the gradual transitions and decreased transition stress values depicted in Figure 6c.

As detailed in $[69,93,99]$, the likelihood $p_{A \pm}$ that austenite will transform to $M^{ \pm}$and the likelihoods $p_{ \pm}$that $M^{ \pm}$will transform either to austenite or the other martensite variant are given by

$$
\begin{aligned}
& p_{A \pm}(\sigma, T)=\sqrt{\frac{k T}{2 \pi \nu V^{2 / 3}}} \frac{e^{-G\left( \pm \varepsilon_{A}, \sigma, T\right) V / k T}}{\int_{\chi_{A}} e^{-G(\varepsilon, \sigma, T) V / k T} d \varepsilon} \\
& p_{ \pm}(\sigma, T)=\sqrt{\frac{k T}{2 \pi \nu V^{2 / 3}}} \frac{e^{-G\left( \pm \varepsilon_{M}, \sigma, T\right) V / k T}}{\int_{\chi_{M^{ \pm}}} e^{-G(\varepsilon, \sigma, T) V / k T} d \varepsilon}
\end{aligned}
$$

where $\chi_{A}(T)=\left(-\varepsilon_{A}(T), \varepsilon_{A}(T)\right), \chi_{M^{+}}(T)=\left(\varepsilon_{M}(T), \infty\right)$ and $\chi_{M^{-}}(T)=\left(-\infty,-\varepsilon_{M}(T)\right)$ respectively denote regions over which austenite, $M^{+}$and $M^{-}$are stable. Moreover, the expected strains due to austenite and $M \pm$ variants are given by

$$
\begin{aligned}
& \left\langle\varepsilon_{-}\right\rangle=\int_{\chi_{M^{-}}} \varepsilon \mu(G(\varepsilon, \sigma, T)) d \varepsilon \\
& \left\langle\varepsilon_{+}\right\rangle=\int_{\chi_{M^{+}}} \varepsilon \mu(G(\varepsilon, \sigma, T)) d \varepsilon \\
& \left\langle\varepsilon_{A}\right\rangle=\int_{\chi_{A}} \varepsilon \mu(G(\varepsilon, \sigma, T)) d \varepsilon
\end{aligned}
$$

where $\mu$ is given in (8) and the Gibbs energy is specified in (39). The evolution of phase fractions is governed by the rate laws

$$
\begin{aligned}
& \dot{x}_{-}(t)=p_{A-} x_{A}(t)-p_{-} x_{-}(t) \\
& \dot{x}_{+}(t)=p_{A+} x_{A}(t)-p_{+} x_{+}(t) \\
& \dot{x}_{A}(t)=p_{-} x_{-}(t)-p_{A-} x_{A}(t)+p_{+} x_{+}(t)-p_{A+} x_{A}(t)
\end{aligned}
$$

which can be reduced to

$$
\begin{aligned}
& \dot{x}_{-}(t)=-\left(p_{-}+p_{A_{-}}\right) x_{-}(t)-p_{A-} x_{+}(t)+p_{A-} \\
& \dot{x}_{+}(t)=-\left(p_{+}+p_{A+}\right) x_{+}(t)-p_{A+} x_{-}(t)+p_{A+}
\end{aligned}
$$

through the conservation relation (34). To compute the local average strain for the reference volume, we make the assumption that thermal strains are small compared to mechanical strains and retain only the latter component. As discussed in [69], this assumption is valid for bulk materials but may need to be modified for SMA thin films. Under this assumption, the local average strains, in regimes for which thermal activation or relaxation mechanisms are significant, are given by

$$
\bar{\varepsilon}=\left\langle\varepsilon_{-}\right\rangle x_{-}+\left\langle\varepsilon_{+}\right\rangle x_{+}+\left\langle\varepsilon_{A}\right\rangle x_{A}
$$

where $x_{-}, x_{+}$are specified by (49), $x_{A}=1-x_{+}-x_{-}$, and $\left\langle\varepsilon_{-}\right\rangle,\left\langle\varepsilon_{+}\right\rangle$and $\left\langle\varepsilon_{A}\right\rangle$ are defined in (47). 


\subsubsection{Thermal Evolution}

The quantification of thermal processes is analogous to that considered in (15) for ferroelectric compounds and (33) for ferromagnetic materials, and includes convective and conductive mechanisms, Joule heating and heat transduction due to phase transitions. As in Sections 2.1.3 and 2.2.3, we let $h_{c}, T_{E}, \rho_{\alpha}^{e}, c_{\alpha}, \Omega, \mathcal{A}, \mathcal{M}, \lambda$ and $\ell$ respectively denote the convection coefficient, temperature of the surrounding environment, phase-dependent electric resistivity, phase-dependent specific heat, SMA surface area, SMA cross-sectional area, the mass of the SMA, the thermal conductivity of the surrounding medium and the length of any conduction paths. An energy balance then yields the differential equation

$$
\mathcal{M} \bar{c}(t) \dot{T}(t)=-\Omega\left[h_{c}+\lambda / \ell\right]\left[T-T_{e}(t)\right]+J(t)-\sum_{\alpha} h_{\alpha} \dot{x}_{\alpha}
$$

which quantified the temperature evolution (see $[69,93,98,99]$ ). The first term on the right hand side characterizes heat exchanged with the surrounding environment through convection and conduction in a manner analogous to (15) and (33) for ferroelectric and ferromagnetic materials. For an input current $I(t)$, the relation

$$
J(t)=\bar{\rho}^{e}(t) \frac{I^{2}(t)}{\mathcal{A}}
$$

where $\bar{\rho}^{e}(t)=\sum_{\alpha} \rho_{\alpha}^{e} x_{\alpha}(t)$ is the average resistivity per unit length, quantifies heat generated through Joule heating. The final term in (51) accounts for heat generated or lost during phase transformations. As discussed in [69], the specific enthalpies $h_{\alpha}$ have the form

$$
h_{\alpha}=g_{\alpha}+T s_{\alpha}
$$

where $g_{\alpha}$ are local minima of the Gibbs relation (39) and $s_{\alpha}$ denotes the specific entropies defined in (38). Finally, the average specific heat is given by $\bar{c}(t)=\sum_{\alpha} c_{\alpha} x_{\alpha}(t)$. We note that $\bar{c}$ and $\bar{\rho}^{e}$ are assumed to be constant in the ferroelectric and ferromagnetic relations whereas their time-dependence in (51) and (52) reflects their dependence on evolving phase distributions in shape memory alloys.

\subsection{Unified Mesoscopic Models}

In Section 2.1, 2.2 and 2.3, we summarized the development of nonlinear hysteresis models for ferroelectric, ferromagnetic and ferroelastic compounds, respectively. We discuss here the unified nature of the modeling framework and indicate the degree to which the three models can be consolidated through common notation.

As noted in the introduction to this section, the polarization $P$, magnetization $M$, and strain $\varepsilon$ can all be represented by the order parameter $e$ whereas the electric field $E$, magnetic field $H$ and stress $\sigma$ can be respectively represented by the external field $\varphi$ which is thermodynamically conjugate to $e$. The definitions of subscripted parameters follow the conventions established in previous subsections (e.g., $e_{I}=P_{I}$ or $M_{I}$ ). In all definitions, the Boltzmann probability $\mu(G)$ is specified by (8) and domains of integration are defined by $\chi_{+}=\left(e_{I}, \infty\right), \chi_{-}=\left(-\infty,-e_{I}\right), \chi_{A}=$ $\left(-\varepsilon_{A}, \varepsilon_{A}\right), \chi_{M^{+}}=\left(\varepsilon_{M}, \infty\right)$ and $\chi_{M^{-}}=\left(-\infty,-\varepsilon_{M}\right)$.

By employing the order parameter, conjugate fields, and appropriate parameters, the magnetization and polarization models can be completely unified in the absence of phase transitions. The strain model for ferroelastic compounds falls within the same modeling framework but some individual definitions differ slightly due to the inherent phase transitions. While notation can be established to formulate all three models in terms of unified definitions and expressions, the required generality 
obscures rather than clarifies the framework so we omit it here. Finally, we note that the unified mesoscopic models summarized here represent only the fundamental theory and a number of generalizations to this theory are discussed in previous subsections.

\section{Ferroelectric and Ferromagnetic Materials}

Helmholtz and Gibbs Energy Relations:

$$
\begin{aligned}
& \psi(e, T)=\frac{\varphi_{h} e_{s}}{2}\left[1-\left(e / e_{s}\right)^{2}\right]+\frac{\varphi_{h} T}{2 T_{c}}\left[e \ln \left(\frac{e+e_{s}}{e_{s}-e}\right)+e_{s} \ln \left(1-\left(e / e_{s}\right)^{2}\right)\right] \\
& \psi(e)= \begin{cases}\frac{1}{2} \eta\left(e+e_{R}\right)^{2} & , e \leq-e_{I} \\
\frac{1}{2} \eta\left(e-e_{R}\right)^{2} & , e \geq e_{I} \\
\frac{1}{2} \eta\left(e_{I}-e_{R}\right)\left(\frac{e^{2}}{e_{I}}-e_{R}\right) & ,|e|<e_{I}\end{cases} \\
& G(e, \varphi, T)=\psi(e, T)-e \varphi
\end{aligned}
$$

Mesoscopic Model - No Relaxation Mechanisms:

$$
\begin{aligned}
& {\left[\bar{e}\left(\varphi ; \varphi_{c}, \xi\right)\right](t)= \begin{cases}{\left[\bar{e}\left(\varphi ; \varphi_{c}, \xi\right)\right](0)} & , \tau(t)=\emptyset \\
\frac{\varphi}{\eta}-e_{R} & , \tau(t) \neq \emptyset \text { and } \varphi(\max \tau(t))=-\varphi_{c} \\
\frac{\varphi}{\eta}+e_{R} & , \tau(t) \neq \emptyset \text { and } \varphi(\max \tau(t))=\varphi_{c}\end{cases} } \\
& {\left[\bar{e}\left(\varphi ; \varphi_{c}, \xi\right)\right](0)= \begin{cases}\frac{\varphi}{\eta}-e_{R} & , \varphi(0) \leq-\varphi_{c} \\
\xi & ,-\varphi_{c}<\varphi(0)<\varphi_{c} \\
\frac{\varphi}{\eta}+e_{R} & , \varphi(0) \geq \varphi_{c}\end{cases} } \\
& \tau(t)=\left\{t \in\left(0, T_{f}\right] \mid \varphi(t)=-\varphi_{c} \text { or } \varphi(t)=\varphi_{c}\right\}
\end{aligned}
$$

Mesoscopic Model - with Relaxation Mechanisms:

$$
\begin{aligned}
& \bar{e}=x_{+}\left\langle e_{+}\right\rangle+x_{-}\left\langle e_{-}\right\rangle \\
& \left\langle e_{ \pm}\right\rangle=\int_{\chi_{ \pm}} e \mu(G(e, \phi, T)) d e \\
& \dot{x}_{+}=-p_{+-} x_{+}+p_{-+}\left(1-x_{+}\right) \\
& x_{-}=1-x_{+} \\
& p_{ \pm}=\frac{1}{\mathcal{T}(T)} \mu\left(G\left( \pm e_{I}, \phi, T\right)\right)
\end{aligned}
$$

Thermal Evolution:

$$
\bar{c} \mathcal{M} \dot{T}(t)=-\Omega\left[h_{c}+\lambda / \ell\right]\left[T-T_{E}(t)\right]+J(t)-\sum_{\alpha} h_{\alpha} \dot{x}_{\alpha}
$$




\section{Ferroelastic Materials - Austenite and Martensite Phases}

Helmholtz and Gibbs Energy Relations:

$$
\psi(e, T)= \begin{cases}\frac{Y_{M}}{2}\left(e+e_{T}\right)^{2} & , e \leq-e_{M}(T) \\ -\frac{E_{0}(T)}{2}\left(e+e_{0}(T)\right)^{2}+\psi_{0}(T) & , \quad-e_{M}(T)<e<-e_{A}(T) \\ \frac{Y_{A}}{2} e^{2}+\Delta \beta(T) & , \quad|e| \leq e_{A}(T) \\ -\frac{E_{0}(T)}{2}\left(e-e_{0}(T)\right)^{2}+\psi_{0}(T) & , \quad e_{A}(T)<e<e_{M}(T) \\ \frac{Y_{M}}{2}\left(e-e_{T}\right)^{2} & , e \geq e_{M}(T) \\ G(e, \varphi, T)=\psi(e, T)-e \varphi & \end{cases}
$$

Mesoscopic Model - No Relaxation Mechanisms:

$$
\begin{aligned}
& {\left[\bar{e}\left(\varphi ; \varphi_{c}, \xi\right)\right](t)= \begin{cases}{\left[\bar{e}\left(\varphi ; \varphi_{c}, \xi\right)\right](0)} & , \tau(t)=\emptyset \\
\frac{\varphi}{Y_{M}}+e_{T} & , \tau(t) \neq \emptyset \text { and } \varphi(\max \tau(t))=\varphi_{M} \\
\frac{\varphi}{Y_{A}} & , \tau(t) \neq \emptyset \text { and } \varphi(\max \tau(t))=\varphi_{A}\end{cases} } \\
& {\left[\bar{e}\left(\varphi ; \varphi_{c}, \xi\right)\right](0)= \begin{cases}\frac{\varphi}{Y_{A}} & , \varphi(0) \leq \varphi_{M} \\
\xi & , \varphi_{M}<\varphi(0)<\varphi_{A} \\
\frac{\varphi}{Y_{M}}+e_{T} & , \varphi(0) \geq \varphi_{A}\end{cases} } \\
& \tau(t)=\left\{t \in\left(0, T_{f}\right] \mid \varphi(t)=\varphi_{M} \text { or } \varphi(t)=\varphi_{A}\right\}
\end{aligned}
$$

Mesoscopic Model - with Relaxation Mechanisms:

$$
\begin{aligned}
& \bar{e}=x_{+}\left\langle e_{+}\right\rangle+x_{-}\left\langle e_{-}\right\rangle+x_{A}\left\langle e_{A}\right\rangle \\
& \left\langle e_{ \pm}\right\rangle=\int_{\chi_{M^{ \pm}}} e \mu(G(e, \phi, T)) d e, \quad\left\langle e_{A}\right\rangle=\int_{\chi_{A}} e \mu(G(e, \phi, T)) d e \\
& \dot{x}_{-}(t)=-\left(p_{-}+p_{A-}\right) x_{-}(t)-p_{A-} x_{+}(t)+p_{A-} \\
& \dot{x}_{+}(t)=-\left(p_{+}+p_{A+}\right) x_{+}(t)-p_{A+} x_{-}(t)+p_{A+} \\
& x_{A}=1-x_{+}-x_{-} \\
& p_{ \pm}=\frac{1}{\mathcal{T}(T)} \mu\left(G\left( \pm e_{M}, \phi, T\right)\right) \quad, \quad p_{A \pm}=\frac{1}{\mathcal{T}(T)} \mu\left(G\left( \pm e_{A}, \phi, T\right)\right)
\end{aligned}
$$

Thermal Evolution:

$$
\mathcal{M} \bar{c} \dot{T}(t)=-\Omega\left[h_{c}+\lambda / \ell\right]\left[T-T_{E}(t)\right]+J(t)-\sum_{\alpha} h_{\alpha} \dot{x}_{\alpha}
$$




\subsection{Macroscopic Models for Homogeneous, Single Crystal Compounds}

The hysteresis models in Sections 2.1, 2.2 and 2.3 were all developed at the lattice level under the assumption of isotropic, homogeneous material properties throughout the reference volume $V$. This implies that the the weighted exchange energy $\Phi_{0}$ employed in the Helmholtz relations (4) and (22) is constant which in turn implies that the respective bias fields $E_{h}=\frac{N \Phi_{0}}{V P_{s}}$ and $H_{h}=\frac{N \Phi_{0}}{V M_{s}}$

and parameters $\alpha=\frac{E_{h}}{P_{s}}$ and $\alpha=\frac{H_{h}}{M_{s}}$ respectively employed in (17) and (25) are constant. Since $E_{e}=E+\alpha P$ and $H_{e}=H+\alpha M$ represent effective fields, the assumption of uniform lattice properties and homogeneous exchange energy relations implies constant effective fields throughout $V$.

For isotropic, homogeneous, single crystal materials, the local average models (14), (17) or (19) for $\bar{P},(25),(29)$ or (32) for $\bar{M}$, and (43) or (50) for $\bar{\varepsilon}$ can be extended throughout the compounds to provide bulk or macroscopic constitutive relations. Hence they can be employed to quantify single crystal behavior of the type experimentally measured for the ferroelectric material $\mathrm{BaTiO}_{3}$ (e.g., see page 76 of [72]) and the shape memory alloy $\mathrm{CuZnAl}[38,109]$.

The local relations do not, however, accurately quantify the gradual transitions and pre-remanent switching characteristic of nonhomogeneous, polycrystalline compounds with nonuniform effective fields. In the next section, we employ the local average polarization, magnetization and strain relations as kernels when developing stochastic homogenization techniques to incorporate the variability inherent to the majority of ferroic compounds.

\section{Macroscopic Models for Nonhomogeneous Polycrystalline Com- pounds}

The mesoscopic models developed in Section 2 can be directly employed when quantifying the polarization and stress-induced strains in certain single crystal ferroelectric and ferroelastic compounds as well as the magnetization in some uniaxial wires and annealed toroidal specimens [23]. However, the transitions provided by these local models are too steep to accurately characterize hysteresis in general ferroic materials due to variations in the free energy relations produced by material, stress and field nonhomogeneities, nonuniform lattice variations across grain boundaries, and stress and crystalline anisotropies. The introduction of these material attributes into the energy relations requires analysis similar to that employed in micromagnetic models $[12,13,14,32,54]$ or micromechanical theory [43] and typically produces models whose complexity precludes transducer design or realtime control implementation. Alternatively, one can employ local energy relations as kernels from which low-order macroscopic models be derived either through homogenization techniques or the determination of bulk effective parameters through stochastic or empirical means. In this section, we consider certain parameters in the local energy-based relations to be stochastically distributed to reflect variations in the lattice, grain orientations, or exchange energies. This yields low-order macroscopic models in which the majority of parameters can be correlated with measured properties of experimental data. As will be illustrated in Section 4, the models accurately characterize both major and biased minor loop data for a wide range of ferroic compounds.

\subsection{Ferroelectric Materials}

We consider first the construction of macroscopic models for ferroelectric compounds. As illustrated in Figure 7, variations in the lattice due to material nonhomogeneities, impurities, grain boundaries, or polycrystallinity, are manifested as variation in the Helmholtz and Gibbs energies which in turn produce a distribution of fundamental hysterons or hysteresis kernels. To incorporate this variability in a manner which facilitates the construction of low-order macroscopic models, we consider the local 


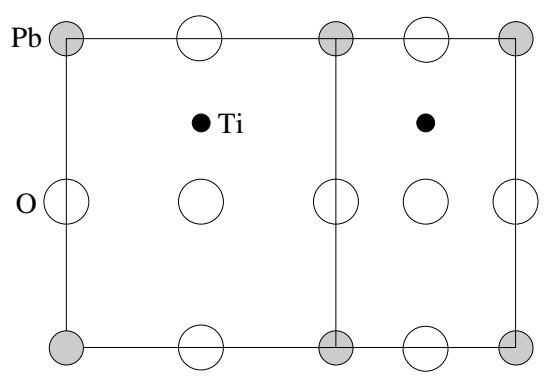

(i)

(a)

(ii)

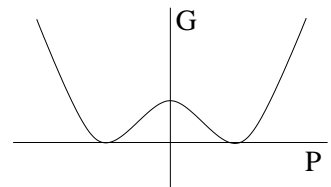

(i)

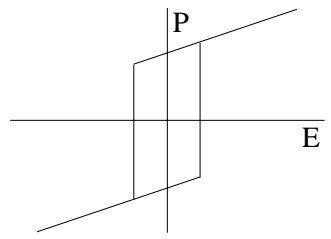

(i)

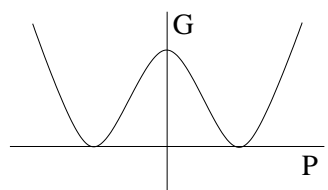

(ii)

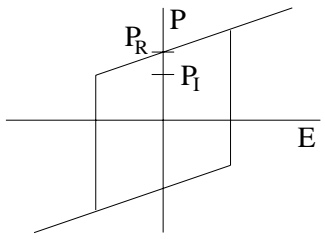

(ii)

(c)

Figure 7. (a) Nonuniform lattice and polycrystalline structure of PZT. (b) Helmholtz energies associated with lattice structures (i) and (ii). (c) Variations in hysteresis kernels due to differing energy profiles.

coercive fields $E_{c}$, given by (6), to be manifestations of an underlying distribution rather than fixed values as assumed for single crystals with uniform lattices. To enforce the physical constraint $E_{c}>0$, we assume that variations in $E_{c}$ can be modeled by a lognormal distribution with the density

$$
f\left(E_{c}\right)=c_{1} e^{-\left[\ln \left(E_{c} / \bar{E}_{c}\right) / 2 c\right]^{2}} .
$$

It is noted in [114] that if the constant $c$ is small compared with $\bar{E}_{c}$, the mean and variance of the distribution have the approximate values

$$
\left\langle E_{c}\right\rangle \approx \bar{E}_{c} \quad, \quad \sigma \approx 2 \bar{E}_{c} c .
$$

The second extension of the local average relations (14), (18) or (21) is the consideration of effective fields in the material. As indicated in [6,70], the applied field $E$ in ferroelectric materials is augmented by fields produced by neighboring dipoles which produces nonhomogeneous effective fields $E_{e}$ in the materials. This produces deviations about the applied field which can produce switching in advance of the remanence point. To incorporate these variations, we consider the effective field to be normally distributed about the applied field with the density

$$
\widetilde{f}\left(E_{e}\right)=c_{2} e^{-\left(E-E_{e}\right)^{2} / b} .
$$


The macroscopic polarization model combines the densities (53) and (55) to yield

$$
[P(E)](t)=C \int_{0}^{\infty} \int_{-\infty}^{\infty}\left[\bar{P}\left(E_{e}+E, E_{c}, \xi\right)\right](t) e^{-E_{e}^{2} / b} e^{-\left[\ln \left(E_{c} / \bar{E}_{c}\right) / 2 c\right]^{2}} d E_{e} d E_{c}
$$

where $\bar{P}$ is specified by (14), (18) or (21) and $\xi$ denotes the initial distribution of dipoles. Discussion concerning the incorporation of lattice variations and variable effective fields in the Ising relation (16) as well as details regarding the approximation of the integrals and implementation of the model are provided in [114].

We note that while the polarization model (56) does incorporate relaxation mechanisms, it does not incorporate dynamic elastic effects and hence should be employed in low frequency regimes. Initial extensions to the free energy relations and the resulting constitutive relations required for constructing fully dynamic models have been investigated in [111]. Finally, we note that the choice of the lognormal and normal densities (53) and (55) is based on their mathematical properties rather than a priori physical arguments. The estimation of general densities, which reflect the measured physical properties of a given material are reported in [107].

\section{$3.2 \quad$ Ferromagnetic Materials}

To accommodate material nonhomogeneities, polycrystallinity and nonuniform effective fields in ferromagnetic materials, we employ lattice and field distributions analogous to their ferroelectric counterparts. Lattice variations can be modeled by assuming that local magnetic coercive fields $H_{c}$ are manifestations of an underlying distribution which, for the models presented here, we assume to have the lognormal density

$$
f\left(H_{c}\right)=c_{1} e^{-\left[\ln \left(H_{c} / \bar{H}_{c}\right) / 2 c\right]^{2}} .
$$

It is noted in [112], where the present modeling framework is used to establish an energy basis for Preisach models, that lognormal densities play a fundamental role in Preisach theory for magnetic materials due to the natural manner through which they enforce positivity in parameter values [31].

To provide additional physical motivation regarding assumed effective field variability and to relate this analysis to previous models, we consider first the local magnetization relation (25), derived from statistical mechanics tenets, with effective fields $H_{e}=H+\alpha M$. As noted in Section 2, the mean field parameter $\alpha$ is given by

$$
\alpha=\frac{H_{h}}{M_{s}}=\frac{N \Phi_{0}}{V M_{s}^{2}}
$$

where $\Phi_{0}$ is the energy required to reorient a single moment in an ordered lattice. Furthermore, it is illustrated in [105] that

$$
\Phi_{0}=2 \xi \mathcal{J}
$$

where $\mathcal{J}$ is the classical exchange integral and $\xi$ denotes the number of neighbors adjacent to a site.

It is implicitly assumed in the mesoscopic theory yielding $\bar{M}$ that $\mathcal{J}$, and hence $\Phi_{0}$, is constant. Thus $\alpha$ in the effective field $H_{e}=H+\alpha M$ is constant, as assumed in the macroscopic hysteresis models developed in $[56,58]$. However, for nonhomogeneous, polycrystalline materials, it is more reasonable to assume that the exchange integral $\mathcal{J}$ is variable rather than constant. At the quantum level, this variability can be incorporated by modeling the overlap of electron wave functions whereas at the macroscopic level, we can incorporate this variability by employing effective fields

$$
H_{e}=H+\alpha M
$$


where $\alpha$ satisfies an underlying distribution. To avoid the implicit dependence on $M$ afforded by (60), one can alternatively model or estimate the distribution of $H_{e}$, and it is this approach that we employ here.

The assumption that the effective field is normally distributed about the applied field $H$ yields the density

$$
\widetilde{f}\left(H_{e}\right)=c_{2} e^{-\left(H-H_{e}\right)^{2} / b}
$$

and macroscopic model

$$
\begin{aligned}
{[M(H)](t) } & =C \int_{0}^{\infty} \int_{-\infty}^{\infty}\left[\bar{M}\left(H_{e}+H, H_{c}, \xi\right)\right](t) e^{-H_{e}^{2} / b} e^{-\left[\ln \left(H_{c} / \bar{H}_{c}\right) / 2 c\right]^{2}} d H_{e} d H_{c} \\
& =C \int_{0}^{\infty} \int_{-\infty}^{\infty} \nu\left(H_{c}, H_{e}\right)\left[\bar{M}\left(H_{e}+H, H_{c}, \xi\right)\right](t) d H_{e} d H_{c}
\end{aligned}
$$

for the bulk magnetization $M$. The kernel, or fundamental hysteron, $\bar{M}$ is given by (27), (29) or (32) and $\xi$ quantifies the initial moment orientation. As with the ferroelectric model, the underlying assumption of lognormal and normal densities for $H_{c}$ and $H_{e}$ can be avoided through the estimation of a general density $\nu$ through techniques analogous to those employed in $[102,117]$ for estimating densities in Preisach models.

Details regarding the formulation and implementation of $(62)$ can be found in $[106,105]$. We note that because the given formulation does not incorporate eddy current losses, it should be restricted to low frequency regimes or transducer configurations which minimize eddy current losses.

\subsection{Ferroelastic Materials}

The effects of polycrystallinity, material nonhomogeneities, and lattice variations across grain boundaries in ferroelastic materials can be incorporated by considering stochastic distributions of the effective stress $\sigma_{e}$ and either the transformation stress $\sigma_{A}$ or relative stress $\delta=\sigma_{A}-\sigma_{M}$ given by (42). Because $\sigma_{A} \geq \sigma_{M}$, as depicted in Figure 6 , variability in $\delta$ can be incorporated through the assumption that $\delta$ is is lognormally distributed with the density function

$$
f(\delta)=c_{1} e^{-[\ln (\delta / \bar{\delta}) / 2 c]^{2}}
$$

as detailed in [69]. Furthermore, it is illustrated in [97], as well as the example of Section 4.3, that for certain ferroelastic compounds, the Laplace relation

$$
f\left(\sigma_{A}\right)=\frac{1}{2 c} e^{-\left|\bar{\sigma}_{A}-\sigma_{A}\right| / c}
$$

can be employed to accurately characterize the distribution of $\sigma_{A}$. Two appropriate choices for the underlying effective field distribution are the normal density relation

$$
\widetilde{f}\left(\sigma_{e}\right)=c_{2} e^{-\left(\sigma-\sigma_{e}\right)^{2} / b}
$$

or the Laplace relation

$$
\widetilde{f}\left(\sigma_{e}\right)=\frac{1}{2 b} e^{-\left|\sigma-\sigma_{e}\right| / b} .
$$

For the densities (63) and (65), this yields the macroscopic relation

$$
[\varepsilon(\sigma, T)](t)=C \int_{0}^{\infty} \int_{-\infty}^{\infty}\left[\bar{\varepsilon}\left(\sigma_{e}+\sigma, \delta, \xi\right)\right](t) e^{-\sigma_{e}^{2} / b} e^{-[\ln (\delta / \bar{\delta}) / 2 c]^{2}} d \sigma_{e} d \delta
$$


quantifying bulk strains due to input stresses and evolving temperatures. The ferroelastic kernel $\bar{\varepsilon}$ is given by (43) or (50) and the multiplicative constant is simply $C=c_{1} \cdot c_{2}$. The choice of the Laplace densities (64) and (66) yields the analogous relation

$$
[\varepsilon(\sigma, T)](t)=C \int_{0}^{\infty} \int_{-\infty}^{\infty}\left[\bar{\varepsilon}\left(\sigma_{e}+\sigma, \delta, \xi\right)\right](t) e^{-\left|\sigma_{e}\right| / b} e^{-\left|\bar{\sigma}_{A}-\sigma_{A}\right| / c} d \sigma_{e} d \sigma_{A}
$$

for $\varepsilon$.

\subsection{Unified Macroscopic Models}

In Section 2.4, we demonstrated that by employing a general order parameter $e=P, M$ or $\varepsilon$ and conjugate field $\varphi=E, H$ or $\sigma$, mesoscopic models characterizing hysteresis in ferroelectric, ferromagnetic and ferroelastic materials could be consolidated to provide a unified, energy-based framework for quantifying hysteresis at the lattice level in ferroic compounds. Here we employ these lattice-level relations as kernels to construct a unified macroscopic framework for modeling hysteresis and constitutive nonlinearities in bulk ferroic materials. This framework can subsequently be employed for material characterization, hybrid transducer design, and unified control design for systems employing ferroelectric, ferromagnetic or ferroelastic actuators and sensors.

We let $\varphi_{c}=E_{c}, H_{c}$ or $\delta$ denote general coercive or relative fields and let $\varphi_{e}=E_{e}, H_{e}$ or $\sigma_{e}$ denote effective fields. We also let $\bar{e}=\bar{P}, \bar{M}$ or $\bar{\varepsilon}$ denote the local average polarization, magnetization, and strain respectively defined in (14) or (21), (27) or (32), and (43) or (50), with general relations for $\bar{e}$ summarized in Section 2.4. Initial dipole, moment and strain configurations are specified by $\xi$.

The hysteresis and constitutive nonlinearities inherent to ferroic compounds can then be quantified by the general relation

$$
[e(\varphi)](t)=C \int_{0}^{\infty} \int_{-\infty}^{\infty}\left[\bar{e}\left(\varphi_{e}+\varphi, \varphi_{c}, \xi\right)\right](t) e^{-\varphi_{e}^{2} / b} e^{-\left[\ln \left(\varphi_{c} / \bar{\varphi}_{c}\right) / 2 c\right]^{2}} d \varphi_{e} d \varphi_{c}
$$

where $C, b, \bar{\varphi}_{c}$ and $c$ are material-dependent parameters. If additional generality is required, one can employ the model

$$
[e(\varphi)](t)=C \int_{0}^{\infty} \int_{-\infty}^{\infty} \nu\left(\varphi_{c}, \varphi_{e}\right)\left[\bar{e}\left(\varphi_{e}+\varphi, \varphi_{c}, \xi\right)\right](t) d \varphi_{e} d \varphi_{c}
$$

where $\nu$ is a general density to be estimated through a least squares fit to data or direct solution techniques analogous to those developed for Preisach models [102]. As indicated in previous discussion, the constitutive models incorporate relaxation mechanisms but do not incorporate general dynamic

effects. Hence (69) and (70) should be employed in low frequency regimes or modes of operation for which dynamic effects are minimal.

\section{Experimental Validation}

To illustrate the performance of the unified modeling framework, we consider the characterization of hysteresis and constitutive nonlinearities in PZT5A, Terfenol-D, and NiTi. In all cases, quasistatic drive conditions were employed to minimize rate dependencies in the PZT and NiTi and eddy current losses in the Terfenol-D. A fundamental step in the model construction entails the estimation of model parameters and in each of the examples, we discuss the manner through which initial parameter estimates can be obtained using properties of the data. 


\subsection{Experimental Validation for PZT5A}

We consider first the characterization of the hysteretic E-P relation for PZT5A. Data was collected from a $1.7 \mathrm{~cm} \times 0.635 \mathrm{~cm} \times 0.0381 \mathrm{~cm}$ PZT wafer at $200 \mathrm{mHz}$ with peak voltages ranging from $600 \mathrm{~V}$ to $1600 \mathrm{~V}$ - corresponding field values can be computed using the relation $E=V / h$ where $h=3.81 \times$ $10^{-4} \mathrm{~m}$ denotes the thickness of the wafer - as shown in Figure 8. The low drive frequency yielded approximately isothermal operating conditions so it was not necessary to incorporate temperature changes through the evolution equation (15).

The field-polarization relation is characterized using the model (56) with the kernel $\bar{P}$ specified by (21) since the focus here is on the quantification of multiple drive levels rather than measurement and characterization of relaxation mechanisms. We point out that similar results have been obtained with the kernel (14) which retains the activation energy mechanisms required to resolve relaxation effects. Regardless of kernel, implementation of (56) requires the approximation of the integrals. This was accomplished using composite Gaussian quadrature over a truncated range of integration chosen to accommodate the decay exhibited by the densities $f$ and $\widetilde{f}$. As illustrated in [114], where details regarding the implementation of the model are provided, convergence was obtained for both integrals using a 4 point Gaussian rule over a composite grid having 20 divisions.

To construct the model, the parameters $P_{R}, \eta, b, \bar{E}_{c}, c$ and $C$ must be estimated using attributes of the data. For the piecewise linear kernel $\bar{P}$ given by (21), $P_{R}$ and $C$ both have the effect of scaling the polarization for a given field input. Hence they can be combined and initially chosen to obtain correct saturation behavior. The slope of the kernel scales through the stochastic homogenization process so measurements of the reciprocal slope $\frac{\partial E}{\partial P}$ provide initial estimates for $\eta$. From (54), it follows that an initial estimate for $\bar{E}_{c}$ is provided by the measured coercive field whereas small values of $c$ are required when characterizing data with a steep transition through coercivity since little variability is exhibited about the mean value $\bar{E}_{c}$. Finally, the parameter $b$ quantifies the variance in the effective field which determines, in part, the degree to which switching occurs before remanence. Materials
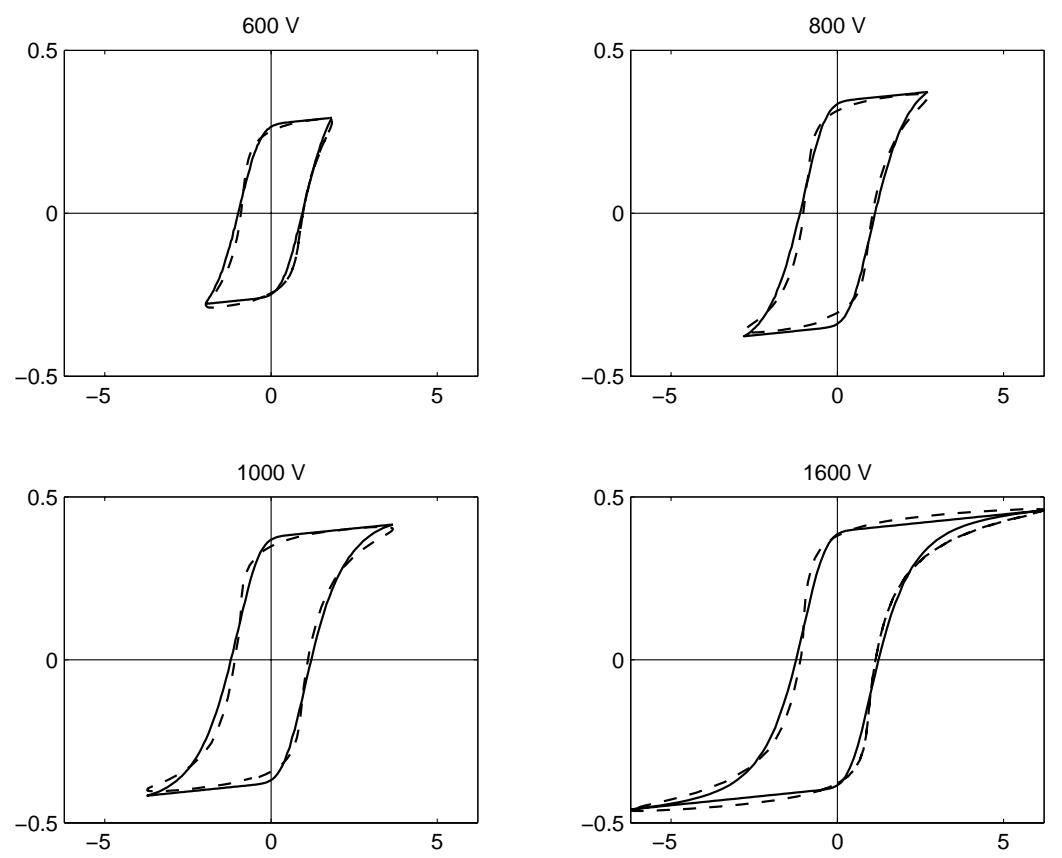

Figure 8. PZT5A data $(---)$ and model predictions (- provided by (56) with the kernel $\bar{P}$ specified by (21). Abscissas: electric field $(\mathrm{MV} / \mathrm{m})$, ordinates: polarization $\left(\mathrm{C} / \mathrm{m}^{2}\right)$. 
with nearly linear $E$ - $P$ relations at remanence yield small values of $b$ whereas large values are required to accommodate significant switching before remanence.

The coercive field for the $1600 \mathrm{~V}$ data is $1.2 \times 10^{6} \mathrm{~V} / \mathrm{m}$ and the slope after reversal at saturation is $3.6 \times 10^{8}$, and these two values were used as initial estimates for $\bar{E}_{c}$ and $\eta$. A least squares fit to the $1600 \mathrm{~V}$ data was used to obtain the final parameter values $P_{R}=0.04 \mathrm{C} / \mathrm{m}^{2}, \bar{E}_{c}=0.866010 \times 10^{6} \mathrm{~V} / \mathrm{m}$, $\eta=9.5 \times 10^{8}, c=0.4272 \mathrm{~V}^{2} / \mathrm{m}^{2}, b=1.9754 \times 10^{11} \mathrm{~V}^{2} / \mathrm{m}^{2}, C=7.9926 \times 10^{-12}$ yielding the model fit illustrated in the final plot of Figure 8. The model with these same values was then used to predict the $E-P$ relations for $600 \mathrm{~V}, 800 \mathrm{~V}$ and $1000 \mathrm{~V}$ inputs. The accuracy of both the high drive level fit and intermediate drive level predictions and the physical nature of parameters attests to the advantages provided by combining an energy-based kernel with stochastic homogenization techniques to accommodate material nonhomogeneities. Further examples illustrating the predictive capabilities of the model for PZT5H and PZT4 compounds, as well as numerical examples illustrating the enforced closure of multiply nested biased minor loops, can be found in [114].

\subsection{Experimental Validation for a Terfenol-D Transducer}

To illustrate the ferromagnetic component of the framework as well as the capability of the model to guarantee closure of biased minor loops in quasistatic operating regimes, we consider the characterization $H-M$ relation for a prototypical Terfenol-D transducer. As illustrated in Figure 9 and detailed in $[17,30]$, Terfenol-D transducers employed for both research development and commercial applications are comprised of a Terfenol-D rod, a surrounding wound wire solenoid, a biasing permanent magnet, and prestress mechanisms. Data collected at $0.2 \mathrm{~Hz}$ under zero prestress, isothermal room temperature conditions is plotted in Figure 10.

The hysteretic and nonlinear relation between $H$ and $M$ is quantified by the model (62) with the kernel $\bar{M}$ specified by (27). The relationships between parameters in the model and attributes of the data are analogous to the ferroelectric case for which details are provided in Section 4.1. To construct the model, the measured coercive field value $H_{c}=6158 \mathrm{~A} / \mathrm{m}$ and reciprocal slope $\frac{d H}{d M}=6.5$ after saturation were used to obtain initial estimates for $\bar{H}_{c}$ and $\eta$. The final parameter values $M_{R}=8.7 \times 10^{4} \mathrm{~A} / \mathrm{m}, \eta=7, H_{c}=2000 \mathrm{~A} / \mathrm{m}, c=0.65 \mathrm{~A}^{2} / \mathrm{m}^{2}, b=5 \times 10^{8} \mathrm{~A}^{2} / \mathrm{m}^{2}$, $C=1.98 \times 10^{-8}$ were then obtained through a least squares fit to the symmetric major loop data yielding the model fit illustrated in Figure 10. Biased, periodic fields were subsequently input to the model, using the same parameter values, to obtain the minor loop predictions, also plotted in Figure 10. We note that when predicting the minor loop responses, the starting magnetization is determined from the symmetric loop fit; hence the accuracy of the minor loops is highly dependent on the accuracy of the symmetric major loop. Additional examples illustrating the attributes of the hysteresis model for ferromagnetic materials can be found in [105, 106].

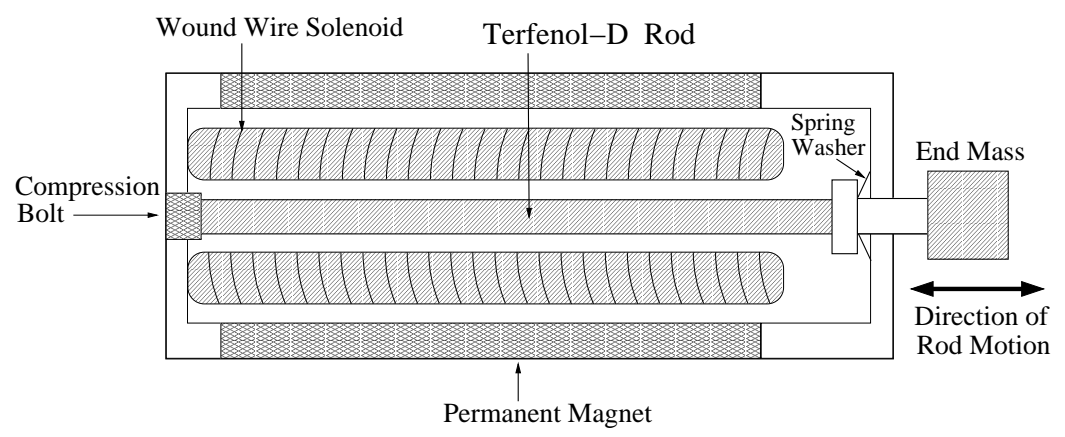

Figure 9. Prototypical Terfenol-D transducer. 


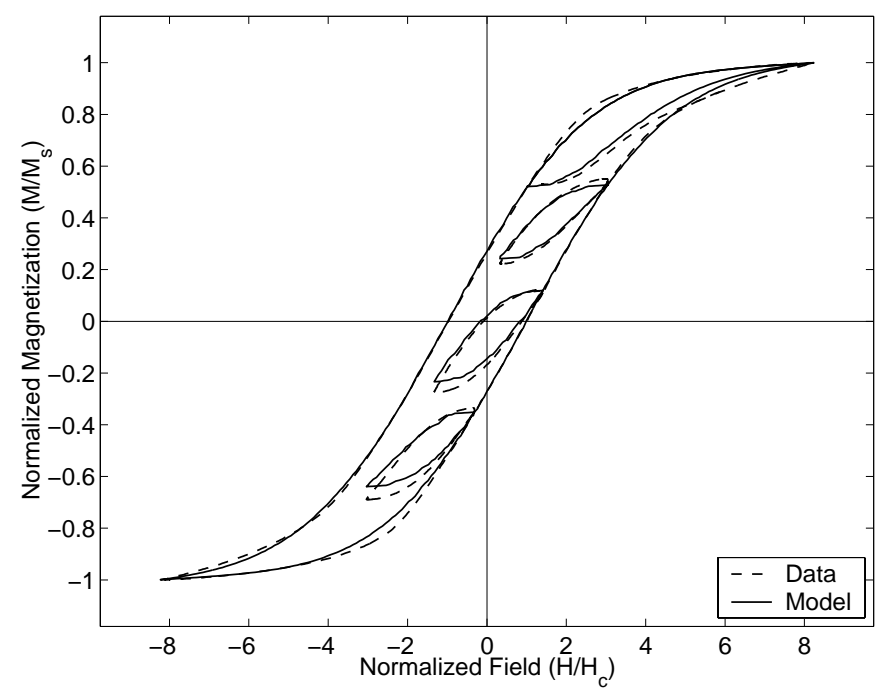

Figure 10. Terfenol-D data and model response provided by (62).

\subsection{Experimental Validation for NiTi}

The final example is taken from [97] and illustrates the characterization of both major and biased minor loops for a pseudoelastic SMA wire in a tensile experiment conducted at room temperature as illustrated in Figure 11.

The wire is a typical NiTi compound (SE508) from Nitinol Devices and Components (NDC) having a diameter of $d=0.5 \mathrm{~mm}$ and length of $\ell=100 \mathrm{~mm}$ which underwent a training procedure of 100 previous loading/unloading cycles. The mesoscopic model parameters include the density $\rho=6400 \mathrm{~kg} \mathrm{~m}^{-3}$, the two Young's moduli $Y_{A}=32 \mathrm{GPa}$ and $Y_{M}=20 \mathrm{GPa}$, and the transformation strain $\varepsilon_{T}=0.038$. The transformation stresses were determined to be $\sigma_{A}\left(T_{L}\right)=430 \mathrm{MPa}$ at $T_{L}=323 \mathrm{~K}$ and $\sigma_{A}\left(T_{U}\right)=660 \mathrm{MPa}$ at $T_{U}=353 \mathrm{~K}$. The width $\delta$ of the hysteresis curve, specified by (42), was taken to be $\delta=100 \mathrm{MPa}$. Since convection mechanisms dominated conduction in the experiments, the heat transfer coefficient was taken to be $h_{c}=200 \mathrm{~W} \mathrm{~m}^{-2} \mathrm{~K}^{-1}$ and the thermal

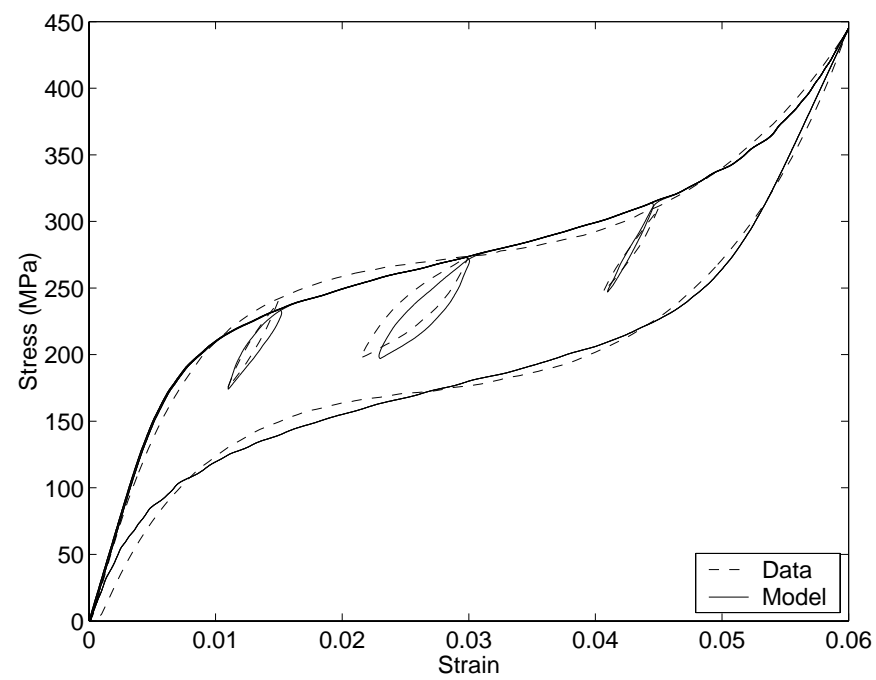

Figure 11. Superelastic NiTi data [94] and model fit provided by (68) with $\bar{\varepsilon}$ specified by (50). 
conductivity was specified as $\lambda=0 \mathrm{~W} \mathrm{~m}^{-1} \mathrm{~K}^{-1}$. The specific heats for austenite and martensite were taken to be equal and having the value of $c_{A}=c_{M}=450 \mathrm{~J} \mathrm{~kg}^{-1} \mathrm{~K}^{-1}$. The relaxation time was chosen to be $\mathcal{T}=10 \mathrm{~ms}$ and the activation volume was specified to be $1 \times 10^{-23} \mathrm{~m}^{3}$. Finally, the macroscopic homogenization was performed using the Laplace distributions (64) and (66) with the parameters $\bar{\sigma}_{A}=273 \mathrm{MPa}$ and $b=c=30 \mathrm{MPa}$.

It is observed that the model accurately characterizes the linearly elastic austenite behavior, the phase transition from austenite to martensite and back again, and the behavior of the nested minor loops. This illustrates the utility of the model for bulk material characterization, transducer design and model-based control design.

\section{Concluding Remarks}

The theory presented here provides a unified framework for modeling hysteresis and constitutive nonlinearities inherent to a broad range of ferroelectric, ferromagnetic, and ferroelastic compounds. At the microscopic scale, the sources of hysteresis vary quite significantly and can roughly be attributed to dipole switching in ferroelectric materials, moment rotation and domain wall losses in ferromagnetic materials, and transitions between austenite and martensite phases in ferroelastic compounds. At the lattice level, however, analogous Helmholtz and Gibbs energy relations can be formulated and employed to construct unified mesoscopic models both in the presence and absence of thermally activated relaxation mechanisms. For homogeneous, single crystal compounds, the mesoscopic relations can be extrapolated to yield macroscopic hysteresis models. To accommodate material nonhomogeneities, polycrystallinity and nonuniform effective fields, stochastic homogenization techniques, based on the assumption that parameters such as coercive, relative and effective fields are manifestations of underlying distributions, are employed to construct macroscopic models. These models provide sufficient accuracy for material characterization but are sufficiently low-order to permit transducer design and model-based control development.

Given the general nature of the framework, it is not surprising that it bears certain similarities with Preisach models and it is illustrated in [112] that it actually provides an energy basis for extended Preisach models - with four fundamental differences. (i) Due to is energy basis, a number of the parameters in the present framework can be directly correlated with properties of the data whereas Preisach parameters must typically be estimated solely through a least squares fit to data. While least squares techniques are often used to fine tune parameters in the present models, the physical correlation provides initial estimates and facilitates model updating to accommodate changing environmental conditions. (ii) The relaxation mechanisms and temperature-dependence are incorporated in the kernels, or hysterons, $\bar{P}, \bar{M}$ and $\bar{\varepsilon}$ rather than in the densities or parameters as is the case for Preisach models. This eliminates the necessity of vector-valued parameters or lookup tables in subsequent control design. (iii) The model guarantees biased minor loop closure in quasistatic operating regimes but does not enforce congruency. Hence it does not require modifications of the type employed in moving Preisach models [31] to accommodate the noncongruency often encountered in measured data. (iv) The model automatically incorporates reversibility through the energy construction of hysterons. This eliminates the modifications required in Preisach models to achieve reversibility in certain drive regimes.

In order for any fixed-temperature, rate independent model to provide an energy basis for classical Preisach representations, it must satisfy the congruency and deletion properties established by Mayergoyz as necessary and sufficient conditions for classical Preisach models [71]. Because the energy-based models do not enforce congruency, they are thus not mathematically equivalent to classical Preisach formulations. However, they do provide an energy basis for the moving Preisach 
models described in [31]. Moreover, use of the kernels (14), (32) or (50) that incorporate relaxation mechanisms through statistical mechanics principles yields macroscopic models which are analogous to the statistically modified Preisach models used to model aftereffects and accommodation in magnetic materials [31]. Analysis quantifying the relationship between this modeling framework and various extended Preisach theories is under present investigation.

From the perspective of model-based control design, this modeling approach is advantageous due to both the accuracy and efficiency provided by the models and the fact that it provides a unified framework for model-based control design. In the context of nonlinear optimal control design, the models have recently been employed when developing control algorithms for piezoceramic transducers [124] and SMA actuators [45, 95] including real-time control implementation as reported in [96]. To permit linear control design, one often employs inverse models as filters to compensate for hysteresis and constitutive nonlinearities so that control inputs to the system are approximately those specified by the control law. Whereas a number of control designs have been developed within this context (e.g., see [24, 26, 42, 44, 73, 74, 75, 103, 117, 118]), all are dependent on having accurate and efficient inverse models. For homogeneous, single crystal transducer compounds, the kernels $\bar{P}, \bar{M}$ or $\bar{\varepsilon}$ can either be directly inverted, or approximately inverted through the solution of complementary differential equations in a manner similar to that detailed for related models in [103, 104]. For nonhomogeneous, polycrystalline transducer compounds, approximate model inverses have been constructed by employing the highly efficient macroscopic models in direct algorithms based on the monotonic relation between external fields and the resulting changes in $P, M$ or $e$ [111]. Robust control designs utilizing approximate inverses constructed in this manner are illustrated for magnetostrictive transducers in [74, 75], and analogous inverse-based control designs for PZT and SMA transducers, and the real-time implementation of resulting control algorithms are under present investigation.

\section{Acknowledgements}

The research of R.C.S. was supported in part through the NSF grant CMS-009764 and in part by the Air Force Office of Scientific Research under the grant AFOSR-F49620-01-1-0107. The research of S.S. was supported in part by the National Science Foundation through the grant DMI-0134464. The research of M.J.D. was provided in part by The Ohio State University through startup funds.

\section{References}

Note: Center for Research in Scientific Computation Technical Reports can be accessed at the web site http://www.ncsu.edu/crsc/reports.html.

[1] M. Achenbach, "A model for an alloy with shape memory," International Journal of Plasticity, 5, pp. 371-395, 1989.

[2] M. Achenbach and I. Müller, "Simulation of material behavior of alloys with shape memory," Archives of Mechanics, 37(6), pp. 573-585, 1985.

[3] A. Adly, I. Mayergoyz and A. Bergqvist, "Preisach modeling of magnetostrictive hysteresis," Journal of Applied Physics, 69(8), pp. 5777-5779, 1991.

[4] S. Aizawa, T. Kakizawa and M. Higashino, "Case studies of smart materials for civil structures," Smart Materials and Structures, 7(5), pp. 617-626, 1998. 
[5] K. Aizu, "Possible species of ferromagnetic, ferroelectric, and ferroelastic crystals," Physical Review B, 2, p. 754, 1970.

[6] J.C. Anderson, Dielectrics, Reinhold Publishing Corporation, New York, 1964.

[7] M. Ashhab, M.V. Salapaka, M. Dahleh and I. Mezic, "Dynamical analysis and control of microcantilevers," Automatica, 1999.

[8] H.T. Banks, A.J. Kurdila G. and G. Webb, "Identification of hysteretic control influence operators representing smart actuators Part I: Formulation," Mathematical Problems in Engineering, 3, pp. 287-328, 1997.

[9] H.T. Banks, A.J. Kurdila and G. Webb, "Identification of hysteretic control influence operators representing smart actuators Part II: Convergent approximations," Journal of Intelligent Material Systems and Structures, 8(6), pp. 536-550, 1997.

[10] J.G. Boyd and D.C. Lagoudas, "Thermomechanical response of shape memory composites," Journal of Intelligent Material Systems and Structures, 5, pp. 333-346, 1994.

[11] M. Brokate and J. Sprekels, Hysteresis and Phase Transitions, Springer-Verlag, New York, 1996.

[12] W.F. Brown, Jr., Magnetostatic Principles in Ferromagnetism, North-Holland Publishing Company, Amsterdam, 1962.

[13] W.F. Brown, Jr., Magnetoelastic Interactions, Springer-Verlag, Berlin, 1966.

[14] W.F. Brown, Jr., "Domain, micromagnetics, and beyond, reminiscences and assessments, Journal of Applied Physics, 49, pp. 1937-1942, 1978.

[15] J.L. Butler, S.C. Butler and A.E. Clark, "Unidirectional magnetostrictive/piezoelectric hybrid transducer," Journal of the Acoustical Society of America, 88, 1990.

[16] S.C. Butler and F.A. Tito, "A broadband hybrid magnetostrictive/piezoelectric transducer array," OCEANS 2000 MTS/IEEE, Vol. 3, pp. 1469-1475, 2000.

[17] F.T. Calkins, R.C. Smith and A.B. Flatau, "An energy-based hysteresis model for magnetostrictive transducers," IEEE Transactions on Magnetics, 36(2), pp. 429-439, 2000.

[18] W. Chen and C.S. Lynch, "A model for simulating polarization switching and AF-F phase changes in ferroelectric ceramics," Journal of Intelligent Material Systems and Structures, 9, pp. 427-431, 1998.

[19] S. Chikazumi, Physics of Ferromagnetism, 2nd Ed., Carendon Press, Oxford, 1997.

[20] H. Chopra, C. Ji and V. Kokorin, "Magnetic-field-induced twin boundary motion in magnetic shape-memory alloys," Physical Review B, 61, pp. 4913-4915, 2000.

[21] A.E. Clark, J.B. Restorff, M. Wun-Fogle, T.A. Lograsso and D.L. Schlagel, "Magnetostrictive properties of Body-Centered-Cubic Fe-Ga and Fe-Ga-Al alloys," IEEE Transactions on Magnetics, 36(5), pp. 3238-3240, 2000.

[22] A.E. Clark, M. Wun-Fogle, J.B. Restorff, T.A. Lograsso, A.R. Ross and D.L. Schlagel, "Magnetostrictive Galfenol/Alfenol single crystal alloys under large compressive stresses," Proceedings of the 7th International Conference on New Actuators, H. Borgmann, Ed., Bremen, Germany, pp. 111, 2000. 
[23] D.J. Craik and R.S. Tebble, Ferromagnetism and Ferromagnetic Domains, John Wiley and Sons, New York, 1965.

[24] D. Croft and S. Devasia, "Vibration compensation for high speed scanning tunneling microscopy," Review of Scientific Instruments, 70(12), pp. 4600-4605, 1999.

[25] D. Croft, G. Shed and S. Devasia, "Creep, hysteresis, and vibration compensation for piezoactuators: Atomic force microscopy application," Journal of Dynamic Systems, Measurement, and Control, 23, pp. 35-43, 2001.

[26] J.M Cruz-Hernandez and V. Hayward, "An approach to reduction of hysteresis in smart materials," Proceedings of the 1998 IEEE International Conference on Robotics and Automation, Leuven, Belgium, pp. 1510-1515, 1998.

[27] B.D. Cullity, Introduction to Magnetic Materials, Addison-Wesley, Reading, MA, 1972.

[28] A. Daniele, S. Salapaka, M.V. Salapaka and M. Dahleh, "Piezoelectric scanners for atomic force microscopes: Design of lateral sensors, identification and control," Proc. of the ACC, San Diego, CA, 1999 pp. 253-257.

[29] M.J. Dapino, R.C. Smith, L.E. Faidley and A.B. Flatau, "A coupled structural-magnetic strain and stress model for magnetostrictive transducers," Journal of Intelligent Material Systems and Structures, 11(2), pp. 134-152, 2000.

[30] M.J. Dapino, R.C. Smith and A.B. Flatau, "A structural-magnetic strain model for magnetostrictive transducers," IEEE Transactions on Magnetics, 36(3), pp. 545-556, 2000.

[31] E. Della Torre, Magnetic Hysteresis, IEEE Press, New York, 1999.

[32] A. DeSimone and R.D. James, "A constrained theory of magnetoelasticity," Journal of the Mechanics and Physics of Solids, 50, pp. 283-320, 2002.

[33] R. DesRoches and M. Delemont, "Seismic retrofit of simply supported bridges using shape memory alloys," Engineering Structures, 24, pp. 325-332, 2002.

[34] M. Dolce and D. Cardone, "Mechanical behaviour of shape memory alloys for seismic applications: 1. Martensite and austenite NiTi bars subjected to torsion," International Journal of Mechanical Sciences, 43, pp. 2631-2656, 2001.

[35] M. Dolce and D. Cardone, "Mechanical behaviour of shape memory alloys for seismic applications: 2. Austenite NiTi wires subjected to tension," International Journal of Mechanical Sciences, 43, pp. 2657-2677, 2001.

[36] P.R. Downey, M.J. Dapino and R.C. Smith, "Analysis of hybrid PMN/Terfenol broadband transducers in mechanical series configuration," Proceedings of the SPIE, Smart Structures and Materials 2003, Volume 5049, pp. 168-179, 2003.

[37] L.E. Faidley, M.J. Dapino, G.N. Washington, R.C. Smith and T.A. Lograsso, "Analytical and experimental issues in Ni-Mn-Ga transducers," Proceedings of the SPIE, Smart Structures and Materials 2003, Volume 5049, pp. 1-12, 2003.

[38] S. Fu, Y. Huo and I. Müller, "Thermodynamics of pseudoelasticiy - An analytic approach," Acta Mechanica, 99, pp. 1-19, 1993. 
[39] J.E. Favelukis, A.S. Lavine and G.P. Carman, "An experimentally validated thermal model of thin-film NiTi," Smart Structures and Materials 1999: Smart Structures and Integrated Systems, Proceedings of the SPIE, Vol. 3668, pt. 1-2, pp. 617-629, 1999.

[40] X. Gao, M. Huang and L.C. Brinson, "A multivariant micromechanical model for SMAs, Part 1: Crystallographic issues for single crystal model," International Journal of Plasticity, 16, pp. 13451369, 2000.

[41] P. Ge and M. Jouaneh, "Modeling hysteresis in piezoceramic actuators," Precision Engineering, 17, pp. 211-221, 1995.

[42] P. Ge and M. Jouaneh, "Tracking control of a piezoceramic actuator," IEEE Transactions on Control Systems Technology, 4(3), pp. 209-216, 1996.

[43] B.C. Goo and C. Lexcellent, "Micromechanics-based modeling of two-way shape memory effect of a single crystalline shape-memory alloy," Acta Materialia, 45(2), pp. 727-737, 1998.

[44] D. Grant and V. Hayward, "Variable structure control of shape memory alloy actuators," IEEE Control Systems Magazine, 17(3), pp. 80-88, 1997.

[45] O. Heintze, S. Seelecke and C. Büskens, "Modeling and control of micro-scale SMA actuators," Proceedings of the SPIE, Smart Structures and Materials 2003, to appear.

[46] K.K. Ho, J.J. Gill, G.P. Carman and P. Jardine, "Fabrication and characterization of thin film NiTi for use as a microbubble for active flow control," Proceedings of the SPIE, Smart Structures and Materials 1999.

[47] H-C. Huang and A.S. Usmani, Finite Element Analysis for Heat Transfer, Springer-Verlag, Berlin, 1994.

[48] L. Huang and H.F. Tiersten, "An analytic description of slow hysteresis in polarized ferroelectric ceramic actuators," Journal of Intelligent Material Systems and Structures, 9, pp. 417-426, 1998.

[49] M. Huang and L.C. Brinson, "A multivariant model for single crystal shape memory alloy behavior," J. Mech. Phys. Solids, 46(8), pp. 1379-1409, 1998.

[50] M. Huang, X. Gao and L.C. Brinson, "A multivariant micromechanical model for SMAs, Part 2: Polycrystal model," International Journal of Plasticity, 16, pp. 1371-1390, 2000.

[51] J.E. Huber, N.A. Fleck, C.M. Landis and R.M. McMeeking, "A constitutive model for ferroelectric polycrystals," Journal of the Mechanics and Physics of Solids, 47, pp. 1663-1697, 1999.

[52] S.C. Hwang and R.M. McMeeking, "The prediction of switching in polycrystalline ferroelectric ceramics," Ferroelectrics, 207, pp. 465-495, 1998.

[53] Y. Ivshin and T.J. Pence, "A thermomechanical model for a one variant shape memory material," Journal of Intelligent Material Systems and Structures, 5, pp. 455-473, 1994.

[54] R.D. James and M. Wuttig, "Magnetostriction of martensite," Philosophical Magazine A, 77(5), pp. 1273-1299, 1998.

[55] H. Janocha and B. Clephas, "Hybrid actuator with piezoelectric and magnetostrictive material," Actuator 96, Proc. 5th International Conference on New Actuators, pp. 304-307, 1996. 
[56] D. Jiles, Introduction to Magnetism and Magnetic Materials, Chapman and Hall, New York, 1991.

[57] D.C. Jiles and D.L. Atherton, "Theory of the magnetisation process in ferromagnetics and its application to the magnetomechanical effect," Journal of Physics D: Applied Physics, 17, pp. 1265-1281, 1984.

[58] D.C. Jiles and D.L. Atherton, "Theory of ferromagnetic hysteresis," Magnetism and Magnetic Materials, 61, pp. 48-60, 1986.

[59] M. Kohl and K.D. Skrobanek, "Linear microactuators based on the shape memory effect," Sensors and Actuators, 70, pp. 104-111, 1998.

[60] M.A. Krasnosel'skiǐ and A.V. Pokrovskiǐ, Systems with Hysteresis, Nauka, Moscow, 1983; Translated by M. Niezgódka, Springer-Verlag, Berlin, 1989.

[61] P. Krulevitch, A.P. Lee, P.B. Ramsey, J.C. Trevino,J. Hamilton and M.A. Northrup, "Thin film shape memory alloy microactuators," Journal of Microelectromechanical Systems, 5(4), pp. 270$282,1996$.

[62] J. Li and G.J. Weng, "A theory of domain switch for the nonlinear behavior of ferroelectrics," Proceedings of the Royal Society of London Series A, 455, pp. 3493-3511, 1999.

[63] F. Liorzou, B. Phelps and D.L. Atherton, "Macroscopic models of magnetization," IEEE Transactions on Magnetics, 36(2), pp. 418-428, 2000.

[64] J. Luan and F.C. Lee, "Design of a high frequency switching amplifier for smart material actuators with improved current mode control," PESC '98 Record, 29th Annual Power Electronics Specialists Conference, Vol. 1, pp. 59-64, 1998.

[65] J.A. Main and E. Garcia, "Design impact of piezoelectric actuator nonlinearities," Journal of Guidance, Control, and Dynamics, 20(2), pp. 327-32, 1997.

[66] J.A. Main and E. Garcia, "Piezoelectric stack actuators and control system design: strategies and pitfalls," Journal of Guidance, Control, and Dynamics. 20(3), pp. 479-485, 1997.

[67] J.A. Main, E. Garcia and D.V. Newton, "Precision position control of piezoelectric actuators using charge feedback," Journal of Guidance, Control, and Dynamics. 18(5), pp. 1068-73, 1995.

[68] J.A. Main, D. Newton, L. Massengil and E. Garcia, "Efficient power amplifiers for piezoelectric applications," Smart Materials and Structures, 5(6), pp. 766-775, 1996.

[69] J.E. Massad, R.C. Smith and G.P. Carman, "A free energy model for thin-film shape memory alloys," Proceedings of the SPIE, Smart Structures and Materials 2003, Volume 5049, pp. 13-23, 2003.

[70] T. Mitsui, I. Tatsuzaki and E. Nakamura, An Introduction to the Physics of Ferroelectrics, Gordon and Breach Science Publishers, New York, 1976.

[71] I.D. Mayergoyz, Mathematical Models of Hysteresis, Springer-Verlag, New York, 1991.

[72] A.J. Moulson and J.M. Herbert, Electroceramic: Materials, Properties, Applications, Chapman and Hill, New York, 1990. 
[73] J.M. Nealis and R.C. Smith, "Partial Inverse Compensation Techniques for Linear Control Design in Magnetostrictive Transducers," Proceedings of the SPIE, Smart Structures and Materials 2001, Volume 4326, pp. 462-473, 2001.

[74] J.M. Nealis and R.C. Smith, "Robust control of a magnetostrictive actuator," Proceedings of the SPIE, Smart Structures and Materials 2003, Volume 5049, pp. 221-232, 2003.

[75] J.M. Nealis and R.C. Smith, " $H_{\infty}$ control of a magnetostrictive actuator," Proc. 42nd IEEE Conference on Decision and Control, submitted.

[76] Newnham, R.E., 1997, "Molecular mechanisms in smart materials," Materials Research Society Bulletin, Volume XXII, No. 5, pp. 20-34.

[77] J.F. Nye, Physical Properties of Crystals: Their Representation by Tensors and Matrices, Oxford, Clarendon Press, 1957.

[78] R. O'Handley, S. Murray, M. Marioni, H. Nembach and S. Allen, "Phenomenology of giant magnetic-field induced strain in ferromagnetic shape-memory materials," Journal of Applied Physics, 87, pp. 4712-4717, 2000.

[79] N. Papenfuß and S. Seelecke, "Simulation and control of SMA actuators," Proceedings of the SPIE, Smart Structures and Materials 1999, Volume 3667, pp. 586-595, 1999.

[80] G. Puglisi and L. Truskinovsky, "Mechanics of a discrete chain with bi-stable elements," Journal of the Mechanics and Physics of Solids, 48, pp. 1-27, 2000.

[81] G. Puglisi and L. Truskinovsky, "Rate independent hysteresis in a bi-stable chain," Journal of the Mechanics and Physics of Solids, 50, pp. 165-187, 2002.

[82] G. Puglisi and L. Truskinovsky, "A mechanism of transformational plasticity," Continuum Mechanics and Thermodynamics, 14(5), pp. 437-457, 2002.

[83] J.B. Restorff, H.T. Savage, A.E. Clark and M. Wun-Fogle, "Preisach modeling of hysteresis in Terfenol-D," Journal of Applied Physics, 67(9), pp. 5016-5018, 1996.

[84] D. Reynaerts and H. van Brussel, "Design aspects of shape memory actuators," Mechatronics, 8, pp. 635-656, 1998.

[85] D. Reynaerts, J. Peirs and H. van Brussel, "Shape memory micro-actuation for a gastro-intestinal intervention system," Sensors and Actuators, 77, pp. 157-166, 1999.

[86] G. Robert, D. Damjanovic and N. Setter, "Preisach modeling of piezoelectric nonlinearity in ferroelectric ceramics," Journal of Applied Physics, 89(9), pp. 5067-5074, 2001.

[87] M.V. Salapaka, H.S. Bergh, J. Lai, A. Majumdar and E. McFarland, "Multimode noise analysis of cantilevers for scanning probe microscopy," Journal of Applied Physics, 81(6), pp. 2480-2487, 1997.

[88] S. Salapaka, A. Sebastian, J.P. Cleveland and M.V. Salapaka, "High bandwidth nano-positioner: A robust control approach," Review of Scientific Instruments, 73(9), pp. 3232-3241, 2002.

[89] G. Schitter, P. Menold, H.F. Knapp, F. Allgower and A. Stemmer, "High performance feedback for fast scanning atomic force microscopes," Review of Scientific Instruments, 72(8), pp. 33203327, 2001. 
[90] A. Sebastion and S. Salapaka, " $H_{\infty}$ loop shaping design for nano-positioning," 2003 American Control Conference, to appear.

[91] S. Seelecke, "Dynamics of a SDOF system with shape memory element," Proceedings of the SPIE, Smart Structures and Materials 2000, Vol. 3992, 2000.

[92] S. Seelecke, "Modeling the dynamic behavior of shape memory alloys," International Journal of Non-Linear Mechanics, 37, pp. 1363-1374, 2002.

[93] S. Seelecke, "A fully coupled thermomechanical model for shape memory alloys, Part I: Theory," Journal of the Mechanics and Physics of Solids, submitted.

[94] S. Seelecke and A. Bergman, "Experimental investigation of the strain-rate dependent inner loop behavior of pseudoelastic NiTi wires," Preprint.

[95] S. Seelecke and C. Büskens, "Optimal control of beam structures by shape memory wires," in OPTI 97, Computer Aided Optimum Design of Structures, Rome, Italy, Sept. 8-10, 1997, (eds., S. Hernandez and C.A. Brebbia), Computational Mechanics Press, Rome, Italy, 1997.

[96] S. Seelecke, C. Büskens, I. Müller and J. Sprekels, "Real-time optimal control of shape memory alloy actuators in smart structures," Online Optimization of Large Scale Systems: State of the Art, (eds., M. Groetschel, S.O. Krumke and J. Rambau), Springer-Verlag, Berlin, 2001.

[97] S. Seelecke and O. Heintze, "A model for the strain-rate dependent inner loop behavior of NiTi," Preprint.

[98] S. Seelecke and O. Kastner, "A fully coupled thermomechanical model for shape memory alloys, Part II: Numerical simulation," Journal of the Mechanics and Physics of Solids, submitted.

[99] S. Seelecke and I. Müller, "Shape memory alloy actuators in smart structures - Modeling and simulation," ASME Applied Mechanics Reviews, 57(1), pp. 23-46, 2004.

[100] A. Shadowitz, The Electromagnetic Field, Dover, New York, 1975.

[101] J.A. Shaw, B-C. Chang, M.A. Iadicola and Y.M Leroy, "Thermodynamics of a 1-D shape memory alloy: modeling, experiments and application," Proceedings of the SPIE, Smart Structures and Materials 2003, Volume 5049, pp. 76-87, 2003.

[102] M.E. Shirley and R. Venkataraman, "On the identification of Preisach measures," Proceedings of the SPIE, Smart Structures and Materials 2003, Vol. 5049, pp. 326-336, 2003.

[103] R.C. Smith, "Inverse compensation for hysteresis in magnetostrictive transducers," Mathematical and Computer Modelling, 33, pp. 285-298, 2001.

[104] R.C. Smith, C. Bouton and R. Zrostlik, "Partial and Full Inverse Compensation for Hysteresis in Smart Material Systems," Proceedings of the 2000 American Control Conference.

[105] R.C. Smith and M.J. Dapino, "A macroscopic free energy theory for ferromagnetic hysteresis," IEEE Transactions on Magnetics, to be submitted.

[106] R.C. Smith, M.J. Dapino and S. Seelecke, "A free energy model for hysteresis in magnetostrictive transducers," Journal of Applied Physics, 93(1), pp. 458-466, 2003. 
[107] R.C. Smith, A. Hatch, S. Seelecke, B. Mukherjee, S. Lui and J. Smith, "The identification of general densities in energy-based hysteresis models for ferroelectric compounds," preprint.

[108] R.C. Smith and C.L. Hom, "Domain wall theory for ferroelectric hysteresis," Journal of Intelligent Material Systems and Structures, 10(3), pp. 195-213, 1999.

[109] R.C. Smith and J.E. Massad, "A unified methodology for modeling hysteresis in ferroic materials," Proceedings of the 2001 ASME Design Engineering Technical Conferences and Computers and Information in Engineering Conference, Vol 6, Pt B, pp. 1389-1398, 2001.

[110] R.C. Smith and Z. Ounaies, "A domain wall model for hysteresis in piezoelectric materials," Journal of Intelligent Material Systems and Structures, 11(1), pp. 62-79, 2000.

[111] R.C. Smith, M.V. Salapaka, A. Hatch, J. Smith and T. De, "Model development and inverse compensator design for high speed nanopositioning," Proc. 41st IEEE Conf. Dec. and Control, 2002, Las Vegas, NV.

[112] R.C. Smith and S. Seelecke, "An energy formulation for Preisach models," Proceedings of the SPIE, Smart Structures and Materials 2002, Volume 4693, pp. 173-182, 2002.

[113] R.C. Smith, S. Seelecke and Z. Ounaies, "A free energy model for piezoceramic materials," Proceedings of the SPIE, Smart Structures and Materials 2002, Volume 4693, pp. 183-190, 2002.

[114] R.C. Smith, S. Seelecke, Z. Ounaies and J. Smith, "A free energy model for hysteresis in ferroelectric materials," Journal of Intelligent Material Systems and Structures, 14(11), pp. 719739, 2003.

[115] A.N. Soukhojak and Y.-M. Chiang "Generalized rheology of active materials," Journal of Applied Physics, 88(11), pp. 6902-6909, 2000.

[116] A. Sozinov, A.A. Likhachev, N. Lanska and K. Ullakko, "Giant magnetic-field induced strain in NiMnGa seven-layered martensite phase," Applied Physics Letters, 80, pp. 1746-1749, 2002.

[117] X. Tan, R. Venkataraman and P.S. Krishnaprasad, "Control of hysteresis: Theory and experimental results," Smart Structures and Materials 2001, Modeling, Signal Processing and Control in Smart Structures, SPIE Vol. 4326, pp. 101-112, 2001.

[118] G. Tao and P.V. Kokotović, Adaptive Control of Systems with Actuator and Sensor Nonlinearities, John Wiley and Sons, New York, 1996.

[119] A. Visintin, Differential Models of Hysteresis, Springer-Verlag, Berlin, 1994.

[120] V.K. Wadhawan, Introduction to Ferroic Materials, Gordon and Breach Science Publishers, Amsterdam, 2000.

[121] K. Wilde, P. Gardoni and Y. Fujino, " Base isolation system with shape memory alloy device for elevated highway bridges," Engineering Structures, 22, pp. 222-229, 2000.

[122] J. Woolman, "Effect of atomic composition on the mechanical properties of thin film pseudoelastic nickel titanium," Master's Thesis, Mechanical and Aerospace Engineering Department, UCLA, in preparation. 
[123] X.D. Zhang and C.A. Rogers, "A macroscopic phenomenological formulation for coupled electromechanical effects in piezoelectricity," Journal of Intelligent Material Systems and Structures, 4, pp. 307-316, 1993.

[124] J. Zhong, S. Seelecke, R.C. Smith and C. Büskens, "Optimal control of piezoceramic actuators," Proceedings of the SPIE, Smart Structures and Materials 2003, Volume 5049, pp. 264-274, 2003. 